\title{
Exponential Stability of Neutral Stochastic Functional Differential Equations with Two-Time-Scale Markovian Switching
}

\author{
Junhao Hu and Zhiying Xu \\ College of Mathematics and Statistics, South-Central University for Nationalities, Wuhan 430074, China \\ Correspondence should be addressed to Junhao Hu; junhaohu74@gmail.com
}

Received 22 December 2013; Accepted 16 January 2014; Published 16 March 2014

Academic Editor: Weihai Zhang

Copyright (C) $2014 \mathrm{~J}$. Hu and Z. Xu. This is an open access article distributed under the Creative Commons Attribution License, which permits unrestricted use, distribution, and reproduction in any medium, provided the original work is properly cited.

We develop exponential stability of neutral stochastic functional differential equations with two-time-scale Markovian switching modeled by a continuous-time Markov chain which has a large state space. To overcome the computational effort and the complexity, we split the large-scale system into several classes and lump the states in each class into one class by the different states of changes of the subsystems; then, we give a limit system to effectively "replace" the large-scale system. Under suitable conditions, using the stability of the limit system as a bridge, the desired asymptotic properties of the large-scale system with Brownian motion and Poisson jump are obtained by utilizing perturbed Lyapunov function methods and Razumikhin-type criteria. Two examples are provided to demonstrate our results.

\section{Introduction}

In many practical dynamical systems such as neural networks, computer aided design, population ecology, chemical process simulation, and automatic control, stochastic differential equations represent the class of important dynamics (see [1-4]). During the recent several years, the asymptotic properties of neutral stochastic functional differential equations have been investigated by many authors (see [5-14]). Mao $[10,11]$ gave the exponential stability of neutral stochastic functional differential equations by using the Razumikhintype theorems. Zhou and $\mathrm{Hu}$ [14] used the same argument to discuss the exponential stability in pth moment of neutral stochastic functional differential equations and neutral stochastic functional differential equations with Markovian switching. Wu et al. [13] examined the almost sure robust stability of nonlinear neutral stochastic functional differential equations with infinite delay, including the exponential stability and the polynomial stability. Song and Shen [12] investigated the asymptotic behavior of neutral stochastic functional differential equations under the more general conditions than the classical linear growth condition. Chen et al. [5] considered the exponential stability in mean square moment of mild solution for impulsive neutral stochastic partial functional differential equations by employing the inequality technique. The attraction and quasi-invariant sets of neutral stochastic partial functional differential equations were also studied in the recent paper [9].

In this paper, we will consider neutral stochastic functional differential equations with two-time-scale Markovian switching modeled by a continuous-time Markov chain which has a large state space. The computational effort and the complexity become a real concern. To overcome the difficulties, we have devoted much effort to the modeling and analysis of such systems, in which one of the main ideas is to split a large-scale system into several classes and lump the states in each class into one state (see [3, 15-21]). Khasminskii et al. for the first time established the asymptotic properties of the Markov chain $r^{\varepsilon}(\cdot)$ by introducing a small parameter $\varepsilon>0$ (see [22]). Yin and Zhang developed the method in their book [4] that a complicated system can be replaced by the corresponding limit system that has a much simpler structure. Motivated by the papers [16, 21], under suitable conditions, using the stability of the limit system as a bridge, we will study the exponential stability of neutral stochastic functional differential equations with Brownian 
motion and Poisson jump by utilizing perturbed Lyapunov function methods and Razumikhin-type criteria.

The remainder of this paper is organized as follows. In Section 2, we introduce some notations and notions needed in our investigation. In Section 3, we state our main results, that is, exponential stability of neutral stochastic functional differential equations with two-time-scale Markovian switching. The exponential stability for neutral stochastic functional differential equations driven by pure jumps is also discussed in Section 4. Finally, two examples are presented to justify and illustrate applications of the theory in Section 5.

\section{Preliminaries}

Throughout this paper, unless otherwise specified, let $\left.\left(\Omega, \mathscr{F}_{,}, \mathscr{F}_{t}\right\}_{t \geq 0}, \mathbb{P}\right)$ be a complete probability space with a filtration $\left\{\mathscr{F}_{t}\right\}_{t \geq 0}$ satisfying the usual conditions (i.e., it is increasing and right continuous and $\mathscr{F}_{0}$ contains all $\mathbb{P}$ null sets). Let $W(t)=\left(W_{1}(t), \ldots, W_{m}(t)\right)^{T}$ be an $m$ dimensional Brownian motion defined on the probability space. For $\tau>0$, let $C\left([-\tau, 0] ; \mathbb{R}^{n}\right)$ denote the family of continuous functions $\varphi$ from $[-\tau, 0]$ to $\mathbb{R}^{n}$ with norm $\|\varphi\|=$ $\sup _{-\tau \leq \theta \leq 0}|\varphi(\theta)|$, where $|\cdot|$ is the Euclidean norm in $\mathbb{R}^{n}$. If $A$ is a vector or matrix, its transpose is denoted by $A^{T}$, while its trace norm is denoted by $|A|=\sqrt{\operatorname{trace}\left(A^{T} A\right)}$. Denote by $C_{\mathscr{F}_{0}}^{b}\left([-\tau, 0] ; \mathbb{R}^{n}\right)$ the family of all $\mathscr{F}_{0}$ measurable and bounded $C\left([-\tau, 0] ; \mathbb{R}^{n}\right)$-valued random variables. For $p>0$ and $t \geq 0$, denote by $L_{\mathscr{F}_{t}}^{p}\left([-\tau, 0] ; \mathbb{R}^{n}\right)$ the family of all $\mathscr{F}_{t}$-measurable $C\left([-\tau, 0] ; \mathbb{R}^{n}\right)$-valued random variables $\phi=\{\phi(\theta):-\tau \leq \theta \leq 0\}$ such that $\sup _{-\tau \leq \theta \leq 0} E|\phi(\theta)|^{p}<\infty$. We will denote the indicator function of a set $G$ by $I_{G}$.

Consider an $n$-dimensional neutral stochastic functional differential equation with Markovian switching as follows:

$$
\begin{aligned}
d & {\left[x(t)-D\left(x_{t}, r(t)\right)\right] } \\
& =f\left(x_{t}, t, r(t)\right) d t+g\left(x_{t}, t, r(t)\right) d w(t),
\end{aligned}
$$

on $t \geq 0$ with initial data $x_{0}=\xi \in C\left([-\tau, 0] ; \mathbb{R}^{n}\right)$ and $x_{t}=$ $x(t+\theta):-\tau \leq \theta \leq 0$, which is regarded as a $C\left([-\tau, 0] ; \mathbb{R}^{n}\right)-$ valued stochastic process. Moreover, $f: C\left([-\tau, 0] ; \mathbb{R}^{n}\right) \times$ $\mathbb{R}_{+} \times \mathbb{S} \rightarrow \mathbb{R}^{n}, g: C\left([-\tau, 0] ; \mathbb{R}^{n}\right) \times \mathbb{R}_{+} \times \mathbb{S} \rightarrow \mathbb{R}^{n \times m}$, $D: C\left([-\tau, 0] ; \mathbb{R}^{n}\right) \times \mathbb{S} \rightarrow \mathbb{R}^{n}$.

Let $r(t)(t \geq 0)$ be a right-continuous Markov chain on the probability space taking values in a finite state space $\mathbb{S}=$ $\{1,2, \ldots, M\}$ with generator $\Gamma=\left(\gamma_{i j}\right)_{M \times M}$ given by

$$
\mathbb{P}\{r(t+\Delta)=j \mid r(t)=i\}= \begin{cases}\gamma_{i j} \Delta+\circ(\Delta), & \text { if } i \neq j, \\ 1+\gamma_{i i} \Delta+\circ(\Delta), & \text { if } i=j\end{cases}
$$

where $\Delta>0$. Here, $\gamma_{i j} \geq 0$ is the transition rate from $i$ to $j$ if $i \neq j$, while $\gamma_{i i}=-\sum_{i \neq j} \gamma_{i j}$.

We assume the Markov $r(\cdot)$ is independent of the Brownian motion $W(\cdot)$. It is well known that almost every sample path $r(\cdot)$ is a right-continuous step function with finite number of simple jumps in any finite subinterval of $\mathbb{R}_{+}:=$ $[0, \infty)$. As a standing hypothesis, we assume that the Markov chain is irreducible. This is equivalent to the condition that, for any $i, j \in \mathbb{S}$, we can find $i_{1}, i_{2}, \ldots, i_{k} \in \mathbb{S}$, such that

$$
\gamma_{i, i_{1}} \gamma_{i_{1}, i_{2}} \cdots \gamma_{i_{k}, j}>0
$$

Then, $\Gamma$ always has an eigenvalue 0 . The algebraic interpretation of irreducibility is $\operatorname{rank}(\Gamma)=M-1$. Under this condition, the Markov chain has a unique stationary distribution $\pi \Gamma=0$, subject to $\sum_{j=1}^{M} \pi_{j}=1$ and $\pi_{j}>0$ for all $j \in \mathbb{S}$. For a realvalued function $\sigma(\cdot)$ defined on $\mathbb{S}$, we define

$$
\Gamma \sigma(\cdot)(i):=\sum_{j \in \mathbb{S}} \gamma_{i j} \sigma(j)=\sum_{j \neq i} \gamma_{i j}(\sigma(j)-\sigma(i)),
$$

for each $i \in \mathbb{S}$.

Let $C^{2,1}\left(\mathbb{R}^{n} \times \mathbb{R}_{+} \times \mathbb{S} ; \mathbb{R}_{+}\right)$denote the family of all nonnegative functions $V(x, t, i)$ on $\mathbb{R}^{n} \times \mathbb{R}_{+} \times \mathbb{S}$, which are continuously twice differentiable in $x$ and once differentiable in $t$. If $V(x, t, i) \in C^{2,1}\left(\mathbb{R}^{n} \times \mathbb{R}_{+} \times \mathbb{S} ; \mathbb{R}_{+}\right)$, define an operator $\mathscr{L} V$ from $C\left([-\tau, 0] ; \mathbb{R}^{n}\right) \times \mathbb{R}_{+} \times \mathbb{S}$ to $\mathbb{R}$ by

$$
\begin{aligned}
& \mathscr{L} V(\varphi, t, i)= V_{t}(\varphi(0)-D(\varphi, i), t, i) \\
&+ V_{x}(\varphi(0)-D(\varphi, i), t, i) f(\varphi, t, i) \\
&+ \frac{1}{2} \operatorname{trace}\left[g^{T}(\varphi, t, i)\right. \\
& \times V_{x x}(\varphi(0)-D(\varphi, i), t, i) \\
&\times g(\varphi, t, i)] \\
&+\sum_{j=1}^{l} \gamma_{i j} V(\varphi(0)-D(\varphi, j), t, j),
\end{aligned}
$$

where

$$
\begin{gathered}
\varphi \in C\left([-\tau, 0] ; \mathbb{R}^{n}\right), \quad V_{t}=\frac{\partial V(x, t, i)}{\partial t}, \\
V_{x}=\left(\frac{\partial V(x, t, i)}{\partial x_{1}}, \frac{\partial V(x, t, i)}{\partial x_{2}}, \ldots, \frac{\partial V(x, t, i)}{\partial x_{n}}\right), \\
V_{x x}=\left(\frac{\partial^{2} V(x, t, i)}{\partial x_{i} \partial x_{j}}\right)_{n \times n} .
\end{gathered}
$$

For a parameter $\varepsilon>0$, we rewrite the Markov chain $r(t)$ as $r^{\varepsilon}(t)$ and the generator $\Gamma$ as $\Gamma^{\mathcal{E}}$. $\Gamma^{\mathcal{E}}$ is given by

$$
\Gamma^{\mathcal{\varepsilon}}=\frac{1}{\mathcal{\varepsilon}} \bar{\Gamma}+\widehat{\Gamma}
$$

where $\bar{\Gamma} / \varepsilon$ represents the fast varying motions and $\widehat{\Gamma}$ represents the slowly changing dynamics. Set $\Gamma^{\mathcal{E}}=\left(\gamma_{i j}^{\varepsilon}\right)_{M \times M}$, $\bar{\Gamma}=\left(\bar{\gamma}_{i j}\right)_{M \times M}$, and $\widehat{\Gamma}=\left(\widehat{\gamma}_{i j}\right)_{M \times M}$. For the sake of simplicity, suppose that

$$
\mathbb{S}=\mathbb{S}^{1} \cup \mathbb{S}^{2} \cup \cdots \cup \mathbb{S}^{l}
$$

with $\mathbb{S}^{k}=\left\{s_{k 1}, \ldots, s_{k M_{k}}\right\}, M=M_{1}+M_{2}+\cdots+M_{l}$, and

$$
\bar{\Gamma}=\operatorname{diag}\left(\bar{\Gamma}^{1}, \ldots, \bar{\Gamma}^{l}\right),
$$


where $\bar{\Gamma}^{k}$ is a generator of a Markov chain taking values in $\mathbb{S}^{k}$, for every $k \in\{1, \ldots, l\}$.

We give the first assumption as follows.

Assumption 1. For each $k \in\{1, \ldots, l\}, \bar{\Gamma}^{k}$ is irreducible.

In order to emphasize the effect of the fast switching, (1) can be given by

$$
\begin{array}{r}
d\left[x^{\varepsilon}-D\left(x_{t}^{\varepsilon}, r^{\varepsilon}(t)\right)\right] \\
=f\left(x_{t}^{\varepsilon}, t, r^{\varepsilon}(t)\right) d t+g\left(x_{t}^{\varepsilon}, t, r^{\varepsilon}(t)\right) d w(t), \\
x_{0}^{\varepsilon}=\xi \in C\left([-\tau, 0] ; \mathbb{R}^{n}\right), \quad r^{\varepsilon}=r_{0} .
\end{array}
$$

To assure the existence and uniqueness of the solution, we give the following standard assumptions.

Assumption 2 (local Lipschitz condition). For each integer $\alpha \geq 1$, there exists a constant $L_{\alpha}>0$ such that

$$
\begin{aligned}
|f(\varphi, t, i)-f(\phi, t, i)| & \vee|g(\varphi, t, i)-g(\phi, t, i)| \\
& \leq L_{\alpha}\|\varphi-\phi\|^{2},
\end{aligned}
$$

for all $i \in \mathbb{S}, t \geq 0$ and those $\varphi, \phi \in C\left([-\tau, 0] ; \mathbb{R}^{n}\right)$ with $\|\varphi\| \vee$ $\|\phi\| \leq \alpha$, and $f(0, t, i) \equiv 0, g(0, t, i) \equiv 0$.

Assumption 3 (linear growth condition). There is an $L>0$, for any $\varphi \in C\left([-\tau, 0] ; \mathbb{R}^{n}\right), t \geq 0, i \in \mathbb{S}$ such that

$$
|f(\varphi, t, i)|^{2} \vee|g(\varphi, t, i)|^{2} \leq L\left(1+\|\varphi\|^{2}\right)
$$

Assumption 4. For all $i \in \mathbb{S}$ and those $\varphi, \phi \in C\left([-\tau, 0] ; \mathbb{R}^{n}\right)$, there is a constant $0<\kappa<1$ such that

$$
\begin{gathered}
|D(\varphi, i)-D(\phi, i)| \leq \kappa\|\varphi-\phi\|^{2}, \\
D(0, i) \equiv 0 .
\end{gathered}
$$

Under Assumptions 2, 3, and 4, (10) has a unique solution denoted by $x^{\varepsilon, \xi, i}(t)$ on $t \geq 0$, where $x^{\varepsilon, \xi, i}$ is dependent on the initial value $(\xi, i)$ (see [8]). Moreover, for every $p>0$ and any compact subset $G$ of $C\left([-\tau, 0] ; \mathbb{R}^{n}\right)$, there is a positive constant $H$ which is independent of $\varepsilon$ such that

$$
\sup _{(\xi, i) \in G \times \mathbb{S}} E\left[\sup _{-\tau \leq s \leq t}\left|x^{\varepsilon, \xi, i}(s)\right|^{p}\right] \leq H, \quad t \geq 0 .
$$

Since the state space of the Markov chain is large, it is too complicated to deal with directly. We need to analyse the limit equation of (10). To continue, make all the states in each $\mathbb{S}^{k}$ into a single state and define an aggregated process $\widetilde{r}^{\varepsilon}(\cdot)$ as

$$
\widetilde{r}^{\varepsilon}(t)=k, \quad \text { if } r^{\varepsilon}(t) \in \mathbb{S}^{k} \text {. }
$$

Denote the state space of $\widetilde{r}^{\varepsilon}(t)$ by $\widetilde{\mathbb{S}}=\{1, \ldots, l\}$, the stationary distribution $\widetilde{\Gamma}^{k}$ by $\mu^{k}=\left(\mu_{1}^{k}, \ldots, \mu_{M_{k}}^{k}\right) \in \mathbb{R}^{1 \times M_{k}}$, and $\bar{\mu}=$ $\operatorname{diag}\left(\mu^{1}, \ldots, \mu^{l}\right) \in \mathbb{R}^{l \times M}$. Define

$$
\widetilde{\Gamma}=\left(\widetilde{\gamma}_{i j}\right)_{l \times l}=\bar{\mu} \widehat{\Gamma} \mathbf{1}
$$

with $\mathbf{1}=\operatorname{diag}\left(\mathbf{1}_{M_{1}}, \ldots, \mathbf{1}_{M_{l}}\right)$ and $\mathbf{1}_{M_{k}}=(1, \ldots, 1)^{T} \in \mathbb{R}^{M_{k} \times 1}$, $k=1, \ldots, l$. It has been known that $\widetilde{r}^{\varepsilon}(\cdot)$ converges weakly to $\tilde{r}(\cdot)$ as $\varepsilon \rightarrow 0$, where $\tilde{r}(\cdot)$ is a continuous-time Markov chain with generator $\widetilde{\Gamma}$ and state space $\widetilde{\mathbb{S}}$ (see [4]). Define

$$
\begin{aligned}
\widetilde{D}(\varphi, k) & =\sum_{j=1}^{M_{k}} \mu_{j}^{k} D\left(\varphi, s_{k j}\right), \\
\tilde{f}(\varphi, t, k) & =\sum_{j=1}^{M_{k}} \mu_{j}^{k} f\left(\varphi, t, s_{k j}\right), \\
\tilde{g}(\varphi, t, k) \tilde{g}^{T}(\varphi, t, k) & =\sum_{j=1}^{M_{k}} \mu_{j}^{k} g\left(\varphi, t, s_{k j}\right) g^{T}\left(\varphi, t, s_{k j}\right),
\end{aligned}
$$

for each $s_{k j} \in \mathbb{S}^{k}$ with $k \in\{1, \ldots, l\}$ and $j \in\left\{1, \ldots, M_{k}\right\}$. It is easy to know that $\widetilde{D}(\varphi, k), \widetilde{f}(\varphi, t, k)$, and $\widetilde{g}(\varphi, t, k)$ are the limits with respect to the stationary distribution of the Markov chain. Consider that, for any $\varphi \neq 0, g\left(\varphi, t, s_{k j}\right) g^{T}\left(\varphi, t, s_{k j}\right)$ are nonnegative definite matrices, so we denote its "square root" of $g\left(\varphi, t, s_{k j}\right) g^{T}\left(\varphi, t, s_{k j}\right)$ by $\tilde{g}(\varphi, t, k)$. For degenerate diffusions, we can see the argument in [23].

The limit equation of (10) is defined as follows:

$$
\begin{aligned}
d & {[\widetilde{\varphi}(0)-\widetilde{D}(\widetilde{\varphi}, \tilde{r}(t))] } \\
& =\widetilde{f}(\widetilde{\varphi}, t, \widetilde{r}(t)) d t+\widetilde{g}(\widetilde{\varphi}, t, \widetilde{r}(t)) d w(t), \\
\tilde{x}_{0} & =\xi, \quad \tilde{r}=\widetilde{r}_{0} .
\end{aligned}
$$

\section{Exponential Stability of NSFDE with Two-Time-Scale Markovian Switching}

In this section, we establish the Razumikhin-type theorem on the exponential stability for (10). Denote by $C^{p}\left(\mathbb{R}^{n} \times\right.$ $\left.\mathbb{R}_{+} \times \widetilde{\mathbb{S}} ; \mathbb{R}_{+}\right)$the family of nonnegative real-valued functions defined on $\mathbb{R}^{n} \times \mathbb{R}_{+} \times \widetilde{\mathbb{S}}$ that are $p$-times continuously differentiable with respect to $x$. At the same time, we need another assumption and a lemma with respect to $V(x, t, i) \epsilon$ $C^{p}\left(\mathbb{R}^{n} \times \mathbb{R}_{+} \times \widetilde{\mathbb{S}} ; \mathbb{R}_{+}\right)$for some $p \geq 4$.

Assumption 5. For each $k \in \widetilde{\mathbb{S}}, V(x, t, i) \rightarrow \infty$ as $|x| \rightarrow$ D. Moreover, $\partial^{p} V(x, t, i)=O(1), \partial^{\imath} V(x, t, i)\left(|x|^{l}+|y|^{l}\right) \leq$ $K\left(|x|^{p}+|y|^{p}+1\right)$ for $1 \leq \iota \leq p-1$, where $\partial^{\imath} V(x, t, i)$ denotes the $\iota$ th derivative of $V(x, t, i)$ with respect to $x$ and $O(y)$ denotes the function of $y$ satisfying $\sup _{y}|O(y)| / y<\infty$.

Lemma 6. Suppose that $p \geq 1$; there is a positive constant $\kappa \in(0,1)$ such that

$$
\begin{array}{r}
\mathbb{E}|D(\varphi, k)|^{p} \leq \kappa^{p} \sup _{-\tau \leq \theta \leq 0} e^{\gamma \theta}\|\varphi\|^{p}, \\
(\varphi, i) \in L_{\mathscr{F}_{t}}^{p}\left([-\tau, 0] ; \mathbb{R}^{n}\right) \times \mathbb{S} .
\end{array}
$$


Then, for any $\xi \in L_{\mathscr{F}_{0}}^{p}\left([-\tau, 0] ; \mathbb{R}^{n}\right)$, the solution for (10) satisfies

$$
\begin{aligned}
& \sup _{-\infty<s \leq t} e^{\gamma s} \mathbb{E}|x(s)|^{p} \\
& \leq \frac{\|\xi\|^{p}}{1-\kappa} \vee \frac{\sup _{0 \leq s \leq t} e^{\gamma s} \mathbb{E}\left|x(s)-D\left(x_{s}, r(s)\right)\right|^{p}}{(1-\kappa)^{p}}, \\
& t \geq 0 .
\end{aligned}
$$

Proof. Note the following elementary inequality:

$$
\begin{array}{r}
(x+y)^{p}=\left(1-\kappa_{1}\right)^{1-p}\left(x^{p}+\kappa_{1}{ }^{1-p} y^{p}\right), \\
\forall x, y \geq 0, \quad \kappa_{1}>0 .
\end{array}
$$

We have from condition (20) that, for any $t \geq 0$,

$$
\begin{aligned}
e^{\gamma t} \mathbb{E}|x(t)|^{p} \leq & e^{\gamma t}\left[(1-\kappa)^{1-p} \mathbb{E}\left|x(t)-D\left(x_{t}, r(t)\right)\right|^{p}\right. \\
& \left.+\kappa^{1-p} \mathbb{E}\left|D\left(x_{t}, r(t)\right)\right|^{p}\right] \\
\leq & (1-\kappa)^{1-p} e^{\gamma t} \mathbb{E}\left|x(t)-D\left(x_{t}, r(t)\right)\right|^{p} \\
& +\kappa e^{\gamma t} \sup _{-\tau \leq \theta \leq 0} e^{\gamma \theta} \mathbb{E}|x(t+\theta)|^{p} \\
\leq & (1-\kappa)^{1-p} \sup _{0 \leq s \leq t} e^{\gamma s} \mathbb{E}\left|x(s)-D\left(x_{s}, r(s)\right)\right|^{p} \\
& +\kappa \sup _{-\tau \leq \theta \leq 0} e^{\gamma(s+\theta)} \mathbb{E}|x(s+\theta)|^{p} \\
\leq & (1-\kappa)^{1-p} \sup _{0 \leq s \leq t} e^{\gamma s} \mathbb{E}\left|x(s)-D\left(x_{s}, r(s)\right)\right|^{p} \\
& +\kappa \sup _{-\infty<s \leq t} e^{\gamma s} \mathbb{E}|x(s)|^{p} .
\end{aligned}
$$

Then,

$$
\begin{aligned}
& \sup _{-\infty<s \leq t} e^{\gamma s} \mathbb{E}|x(s)|^{p} \\
& \leq\left[\sup _{-\tau \leq \theta \leq 0} \mathbb{E}|x(\theta)|^{2}\right] \\
& \vee \\
& \quad\left[(1-\kappa)^{1-p} \sup _{0 \leq s \leq t} e^{\gamma s} \mathbb{E}\left|x(s)-D\left(x_{s}, r(s)\right)\right|^{p}\right. \\
& \left.\quad+\kappa \sup _{-\infty<s \leq t} e^{\gamma s} \mathbb{E}|x(s)|^{p}\right] .
\end{aligned}
$$

Therefore, the desired result holds.

Theorem 7. Let Assumptions 1-4 hold and let $c_{1}, c_{2}, \lambda$, $p$ be all positive numbers and $q>1$. Assume that there exists a function $V(x, t, k) \in C^{p}\left(\mathbb{R}^{n} \times \mathbb{R}_{+} \times \widetilde{\mathbb{S}} ; \mathbb{R}_{+}\right)$satisfying Assumption 5 , such that

$$
c_{1}|x|^{p} \leq V(x, t, k) \leq c_{2}|x|^{p}
$$

for all $(x, t, k) \in \mathbb{R}^{n} \times \mathbb{R}_{+} \times \widetilde{\mathbb{S}}, t \geq 0, k \in \widetilde{\mathbb{S}}$. Consider the following:

$$
\begin{aligned}
& \mathbb{E}|D(\varphi, k)|^{p} \leq \kappa^{p} \sup _{-\tau \leq \theta \leq 0} e^{\nu \theta}\|\varphi\|^{p}, \\
& \kappa=\max \left\{\kappa_{1}, \ldots, \kappa_{k}\right\}, \quad \varphi \in L_{\mathscr{F}_{t}}^{p},
\end{aligned}
$$

for all $t \geq 0,0<\kappa_{\sigma}<1, \sigma=\{1, \ldots, k\}$, and

$$
\begin{aligned}
& \mathbb{E}\left[\max _{k \in \widetilde{\mathbb{S}}} \mathscr{L} V(\varphi, t, k)\right] \\
& \quad \leq-\lambda \mathbb{E}\left[\max _{k \in \widetilde{\mathbb{S}}} V(\varphi(0)-D(\varphi, k), t, k)\right],
\end{aligned}
$$

provided $\varphi=\{\varphi(\theta):-\tau \leq \theta \leq 0\} \in L_{\mathscr{F}_{t}}^{p}\left([-\tau, 0] ; \mathbb{R}^{n}\right)$, satisfying

$$
\begin{aligned}
\mathbb{E} & {\left[\min _{k \in \widetilde{\mathbb{S}}} V(\varphi(\theta), t+\theta, k)\right] } \\
& <q \mathbb{E}\left[\max _{k \in \widetilde{\mathbb{S}}} V(\varphi(0)-D(\varphi, k), t, k)\right],
\end{aligned}
$$

for all $-\tau \leq \theta \leq 0$. Then, for all $\xi \in C_{\mathscr{F}_{0}}^{b}\left([-\tau, 0] ; \mathbb{R}^{n}\right), t \geq 0$,

$$
\limsup _{\varepsilon \rightarrow 0}\left|x^{\varepsilon}(t)\right|^{p} \leq \frac{c_{2}(1+\kappa)^{p}}{c_{1}(1-\kappa)^{p}}\|\xi\|^{p} e^{-v t},
$$

where

$$
\nu=\min \left\{\bar{\gamma}, \frac{1}{\tau} \log \frac{q}{\left(c_{2} / c_{1}\right)(1-\kappa)^{p}}\right\},
$$

$\bar{\gamma}$ being the root of the following equation:

$$
\frac{c_{2}}{c_{1}}(1-\kappa)^{p} e^{\gamma \bar{\tau}}=\lambda .
$$

In other words, the trivial solution of (10) is $p$ th moment exponentially stable and the $p$ th moment Lyapunov exponent is not greater than $-\nu$.

Proof. Let

$$
\widetilde{V}(\varphi, t, j)=\sum_{k=1}^{l} V(\varphi, t, k) I_{\left\{j \in \mathbb{S}^{k}\right\}}=V(\varphi, t, k), \quad \text { if } j \in \mathbb{S}^{k} .
$$

By the definition of $\widetilde{V}$, we know that

$$
\begin{gathered}
\widetilde{V}\left(\varphi^{\varepsilon}, t, r^{\varepsilon}(t)\right)=V\left(\varphi^{\varepsilon}, t, \widetilde{r}^{\varepsilon}(t)\right), \\
\sum_{i=1}^{M} \widetilde{\gamma}_{l i} \widetilde{V}(\varphi, t, i)=\sum_{i=1}^{M} \widetilde{\gamma}_{l i} \sum_{k=1}^{l} V(\varphi, t, k) I_{\left\{i \in \mathbb{S}^{k}\right\}}=0 .
\end{gathered}
$$

Extend $r(t)$ to $[-\tau, 0]$ by setting $r(t)=r(0)$. Recalling the facts that $x(t)$ is continuous for all $-\tau \leq \theta \leq 0$ and $r(t)$ is 
right continuous, it is easy to see that $\mathbb{E} V(x(t), t, r(t))$ is right continuous on $t \geq-\tau$. Let $\gamma \in(0, \nu)$ be arbitrary and define

$$
\begin{gathered}
U(t):=\sup _{-\tau \leq \theta \leq 0}\left[e ^ { \gamma ( t + \theta ) } \mathbb { E } V \left(x^{\varepsilon}(t+\theta)-D\left(x_{t+\theta}^{\varepsilon}, \widetilde{r}^{\varepsilon}(t+\theta)\right),\right.\right. \\
\left.\left.t+\theta, \widetilde{r}^{\varepsilon}(t+\theta)\right)\right] \\
=\sup _{-\tau \leq \theta \leq 0}\left[e ^ { \gamma ( t + \theta ) } \mathbb { E } \widetilde { V } \left(x^{\varepsilon}(t+\theta)-D\left(x_{t+\theta}^{\varepsilon}, r^{\varepsilon}(t+\theta)\right),\right.\right. \\
\left.\left.t+\theta, r^{\varepsilon}(t+\theta)\right)\right],
\end{gathered}
$$

for all $t \geq 0$. We claim that

$$
D^{+} U(t)=\limsup _{h \rightarrow 0+} \frac{U(t+h)-U(t)}{h} \leq 0, \quad \forall t \geq 0 .
$$

Note that, for each $t \geq 0$, either $U(t)>e^{\gamma t} \mathbb{E} V\left(x^{\varepsilon}(t)-\right.$ $\left.D\left(x_{t}^{\varepsilon}, \widetilde{r}^{\varepsilon}(t)\right), t, \widetilde{r}^{\varepsilon}(t)\right)$ or $U(t)=e^{\gamma t} \mathbb{E} V\left(x^{\varepsilon}(t)-D\left(x_{t}^{\varepsilon}, \widetilde{r}^{\varepsilon}(t)\right)\right.$, $\left.t, \widetilde{r}^{\varepsilon}(t)\right)$.

If $U(t)>e^{\gamma t} \mathbb{E} V\left(x^{\varepsilon}(t)-D\left(x_{t}^{\varepsilon}, \widetilde{r}^{\varepsilon}(t)\right), t, \widetilde{r}^{\varepsilon}(t)\right)$, because $\mathbb{E} V(x(t), t, r(t))$ is right continuous on $t \geq-\tau$, it is easy to obtain that, for all $h>0$ sufficiently small, $U(t)>$ $e^{\gamma(t+h)} \mathbb{E} V\left(x^{\varepsilon}(t+h)-D\left(x_{t+h}^{\varepsilon}, \widetilde{r}^{\varepsilon}(t+h)\right), t+h, \widetilde{r}^{\varepsilon}(t+h)\right)$; hence, $U(t+h) \leq U(t)$ and $D^{+} U(t) \leq 0$.

If $U(t)=e^{\gamma t} \mathbb{E} V\left(x^{\varepsilon}(t)-D\left(x_{t}^{\varepsilon}, \widetilde{r}^{\varepsilon}(t)\right), t, \widetilde{r}^{\varepsilon}(t)\right)$, we have

$$
\begin{aligned}
& e^{\gamma(t+\theta)} \mathbb{E} V\left(x^{\varepsilon}(t+\theta)-D\left(x_{t+\theta}, \widetilde{r}^{\varepsilon}(t+\theta)\right), t+\theta, \widetilde{r}^{\varepsilon}(t+\theta)\right) \\
& \quad \leq e^{\gamma t} \mathbb{E} V\left(x^{\varepsilon}(t)-D\left(x_{t}, \widetilde{r}^{\varepsilon}(t)\right), t, \widetilde{r}^{\varepsilon}(t)\right),
\end{aligned}
$$

for all $-\tau \leq \theta \leq 0$.

Then,

$$
\begin{aligned}
\mathbb{E} V & \left(x^{\varepsilon}(t+\theta)-D\left(x_{t+\theta}, \widetilde{r}^{\varepsilon}(t+\theta)\right), t+\theta, \widetilde{r}^{\varepsilon}(t+\theta)\right) \\
& \leq e^{-\gamma \theta} \mathbb{E} V\left(x^{\varepsilon}(t)-D\left(x_{t}, \widetilde{r}^{\varepsilon}(t)\right), t, \widetilde{r}^{\varepsilon}(t)\right) \\
& \leq e^{\gamma \tau} \mathbb{E} V\left(x^{\varepsilon}(t)-D\left(x_{t}, \widetilde{r}^{\varepsilon}(t)\right), t, \widetilde{r}^{\varepsilon}(t)\right),
\end{aligned}
$$

for all $-\tau \leq \theta \leq 0$.

On the other hand, by Lemma 6, we derive

$$
\begin{aligned}
& e^{\gamma(t+\theta)} \mathbb{E} V\left(x^{\varepsilon}(t+\theta), t+\theta, \widetilde{r}^{\varepsilon}(t+\theta)\right) \\
& \quad \leq c_{2} e^{\gamma(t+\theta)} \mathbb{E}\left|x^{\varepsilon}(t+\theta)\right|^{p} \\
& \quad \leq c_{2}(1-\kappa)^{p} \sup _{0 \leq s \leq t} e^{\gamma s} \mathbb{E}\left|x^{\varepsilon}(s)-D\left(x_{s}^{\varepsilon}, \widetilde{r}(s)\right)\right|^{p} \\
& \quad \leq \frac{c_{2}}{c_{1}}(1-\kappa)^{p} \sup _{0 \leq s \leq t} e^{\gamma s} \mathbb{E} V\left(x^{\varepsilon}(s)-D\left(x_{s}^{\varepsilon}, \widetilde{r}(s)\right), s, \widetilde{r}(s)\right) \\
& \quad \leq \frac{c_{2}}{c_{1}}(1-\kappa)^{p} e^{\gamma t} \mathbb{E} V\left(x^{\varepsilon}(t)-D\left(x_{t}^{\varepsilon}, \widetilde{r}(t)\right), t, \widetilde{r}(t)\right) .
\end{aligned}
$$

Then,

$$
\begin{aligned}
\mathbb{E} V & \left(x^{\varepsilon}(t+\theta), t+\theta, \widetilde{r}^{\varepsilon}(t+\theta)\right) \\
& <q \mathbb{E} V\left(\varphi^{\varepsilon}(0)-D\left(\varphi^{\varepsilon}, \widetilde{r}^{\varepsilon}(t)\right), t, \widetilde{r}^{\varepsilon}(t)\right),
\end{aligned}
$$

where $q>\left(c_{2} / c_{1}\right)(1-\kappa)^{p} e^{\gamma \tau}$; that is, $\gamma<(1 / \tau)\left(\log \left(q /\left(c_{2} / c_{1}\right)\right.\right.$ $\left.\left.(1-\kappa)^{p}\right)\right)$.

Consequently, there exists a sufficiently small $\varepsilon_{0}>0$, such that, for any $\varepsilon \in\left(0, \varepsilon_{0}\right)$,

$$
\begin{aligned}
& \mathbb{E}\left[\min _{k \in \widetilde{\mathbb{S}}} V\left(\varphi^{\varepsilon}(\theta), t+\theta, k\right)\right] \\
& <q \mathbb{E}\left[\max _{k \in \widetilde{\mathbb{S}}} V\left(\varphi^{\varepsilon}(0)-D\left(\varphi^{\varepsilon}, k\right), t, k\right)\right],
\end{aligned}
$$

for all $-\tau \leq \theta \leq 0$. Thus,

$$
\begin{aligned}
& \mathbb{E}\left[\max _{k \in \widetilde{\mathbb{S}}} \mathscr{L} V\left(\varphi^{\varepsilon}, t, \widetilde{r}^{\varepsilon}(t)\right)\right] \\
& \quad \leq-\lambda \mathbb{E}\left[\max _{k \in \widetilde{\mathbb{S}}} V\left(\varphi^{\varepsilon}(0)-D\left(\varphi^{\varepsilon}, \widetilde{r}^{\varepsilon}(t)\right), t, \widetilde{r}^{\varepsilon}(t)\right)\right],
\end{aligned}
$$

which implies that

$$
\begin{aligned}
\mathbb{E} & {\left[\mathscr{L} V\left(\varphi^{\varepsilon}, t, \widetilde{r}^{\varepsilon}(t)\right)\right] } \\
& \leq-\lambda \mathbb{E}\left[V\left(\varphi^{\varepsilon}(0)-D\left(\varphi^{\varepsilon}, \widetilde{r}^{\varepsilon}(t)\right), t, \widetilde{r}^{\varepsilon}(t)\right)\right] .
\end{aligned}
$$

By the condition of $\gamma<\nu \leq \lambda$, we get

$$
\begin{aligned}
\mathbb{E} & {\left[\mathscr{L} V\left(\varphi^{\varepsilon}, t, \widetilde{r}^{\varepsilon}(t)\right)\right] } \\
& \leq-\gamma \mathbb{E}\left[V\left(\varphi^{\varepsilon}(0)-D\left(\varphi^{\varepsilon}, \widetilde{r}^{\varepsilon}(t)\right), t, \widetilde{r}^{\varepsilon}(t)\right)\right] .
\end{aligned}
$$

Next, we consider

$$
\begin{aligned}
& U(t+h)-U(t) \\
& =\limsup _{\varepsilon \rightarrow 0}\left[e ^ { \gamma ( t + \theta + h ) } \mathbb { E } \left[V \left(x^{\varepsilon}(t+\theta+h)\right.\right.\right. \\
& -D\left(x_{t+\theta+h}^{\varepsilon}, \widetilde{r}^{\varepsilon}(t+\theta+h)\right), \\
& \left.\left.t+\theta+h, \widetilde{r}^{\varepsilon}(t+\theta+h)\right)\right] \\
& -e^{\gamma(t+\theta)} \mathbb{E}\left[V \left(x^{\varepsilon}(t+\theta)-D\left(x_{t+\theta}^{\varepsilon}, \widetilde{r}^{\varepsilon}(t+\theta)\right),\right.\right. \\
& \left.\left.\left.t+\theta, \widetilde{r}^{\varepsilon}(t+\theta)\right)\right]\right] \\
& =\limsup _{\varepsilon \rightarrow 0} \mathbb{E} \int_{t+\theta}^{t+\theta+h} e^{\gamma s}\left[\mathscr{L} V\left(\varphi^{\varepsilon}, s, \widetilde{r}^{\varepsilon}(s)\right)\right. \\
& +\gamma V\left(\varphi^{\varepsilon}(0)-D\left(\varphi^{\varepsilon}, \widetilde{r}^{\varepsilon}(s)\right),\right. \\
& \left.\left.s, \widetilde{r}^{\varepsilon}(s)\right)\right] d s \\
& =\limsup _{\varepsilon \rightarrow 0} \mathbb{E} \int_{t+\theta}^{t+\theta+h} e^{\gamma s}\left[\mathscr{L} \widetilde{V}\left(\varphi^{\varepsilon}, s, r^{\varepsilon}(s)\right)\right. \\
& +\gamma V\left(\varphi^{\varepsilon}(0)-D\left(\varphi^{\varepsilon}, \widetilde{r}^{\varepsilon}(s)\right),\right. \\
& \left.\left.s, \widetilde{r}^{\varepsilon}(s)\right)\right] d s .
\end{aligned}
$$


By the definition of operator $\mathscr{L}$, we have

$$
\begin{aligned}
& \mathscr{L} \widetilde{V}\left(\varphi^{\varepsilon}, t, r^{\varepsilon}(t)\right) \\
& =\widetilde{V}_{t}\left(\varphi^{\varepsilon}(0)-D\left(\varphi^{\varepsilon}, r^{\varepsilon}(t)\right), t, r^{\varepsilon}(t)\right) \\
& +\widetilde{V}_{x}\left(\varphi^{\varepsilon}(0)-D\left(\varphi^{\varepsilon}, r^{\varepsilon}(t)\right), t, r^{\varepsilon}(t)\right) \\
& \times f\left(\varphi^{\varepsilon}, t, r^{\varepsilon}(t)\right) \\
& +\frac{1}{2} \operatorname{trace}\left[g^{T}\left(\varphi^{\varepsilon}, t, r^{\varepsilon}(t)\right)\right. \\
& \times \widetilde{V}_{x x}\left(\varphi^{\varepsilon}(0)-D\left(\varphi^{\varepsilon}, r^{\varepsilon}(t)\right), t, r^{\varepsilon}(t)\right) \\
& \left.\times g\left(\varphi^{\varepsilon}, t, r^{\varepsilon}(t)\right)\right] \\
& +\sum_{i=1}^{M} \gamma_{r^{\varepsilon}(t) i}^{\varepsilon} \widetilde{V}\left(\varphi^{\varepsilon}(0)-D\left(x_{t}^{\varepsilon}, i\right), t, i\right) \\
& =\widetilde{V}_{t}\left(\varphi^{\varepsilon}(0)-D\left(\varphi^{\varepsilon}, r^{\varepsilon}(t)\right), t, r^{\varepsilon}(t)\right) \\
& +\widetilde{V}_{x}\left(\varphi^{\varepsilon}(0)-D\left(\varphi^{\varepsilon}, r^{\varepsilon}(t)\right), t, r^{\varepsilon}(t)\right) \\
& \times f\left(\varphi^{\varepsilon}, t, r^{\varepsilon}(t)\right) \\
& +\frac{1}{2} \operatorname{trace}\left[g^{T}\left(\varphi^{\varepsilon}, t, r^{\varepsilon}(t)\right)\right. \\
& \times \widetilde{V}_{x x}\left(\varphi^{\varepsilon}(0)-D\left(\varphi^{\varepsilon}, r^{\varepsilon}(t)\right), t, r^{\varepsilon}(t)\right) \\
& \left.\times g\left(\varphi^{\varepsilon}, t, r^{\varepsilon}(t)\right)\right] \\
& +\sum_{i=1}^{M} \widehat{\gamma}_{r^{\varepsilon}(t) i} \widetilde{V}\left(\varphi^{\varepsilon}(0)-D\left(x_{t}^{\varepsilon}, i\right), t, i\right) \\
& =V_{t}\left(\varphi^{\varepsilon}(0)-D\left(\varphi^{\varepsilon}, \widetilde{r}^{\varepsilon}(t)\right), t, \widetilde{r}^{\varepsilon}(t)\right) \\
& +V_{x}\left(\varphi^{\varepsilon}(0)-D\left(\varphi^{\varepsilon}, \widetilde{r}^{\varepsilon}(t)\right), t, \widetilde{r}^{\varepsilon}(t)\right) \\
& \times \tilde{f}\left(\varphi^{\varepsilon}, t, \widetilde{r}^{\varepsilon}(t)\right) \\
& +\frac{1}{2} \operatorname{trace}\left[\tilde{g}^{T}\left(\varphi^{\varepsilon}, t, \widetilde{r}^{\varepsilon}(t)\right)\right. \\
& \times V_{x x}\left(\varphi^{\varepsilon}(0)-D\left(\varphi^{\varepsilon}, \widetilde{r}^{\varepsilon}(t)\right), t, \widetilde{r}^{\varepsilon}(t)\right) \\
& \left.\times \tilde{g}\left(\varphi^{\varepsilon}, t, \widetilde{r}^{\varepsilon}(t)\right)\right] \\
& +\sum_{k=1}^{l} \tilde{\gamma}_{\tilde{r}^{\varepsilon}(t) k}^{\varepsilon} V\left(\varphi^{\varepsilon}(0)-D\left(x_{t}^{\varepsilon}, k\right), t, k\right) \\
& +V_{x}\left(\varphi^{\varepsilon}(0)-D\left(\varphi^{\varepsilon}, \widetilde{r}^{\varepsilon}(t)\right), t, \widetilde{r}^{\varepsilon}(t)\right) \\
& \times\left[f\left(\varphi^{\varepsilon}, t, r^{\varepsilon}(t)\right)-\tilde{f}\left(\varphi^{\varepsilon}, t, \widetilde{r}^{\varepsilon}(t)\right)\right] \\
& +\frac{1}{2} \operatorname{trace}\left[g^{T}\left(\varphi^{\varepsilon}, t, r^{\varepsilon}(t)\right)\right. \\
& \times V_{x x}\left(\varphi^{\varepsilon}(0)-D\left(\varphi^{\varepsilon}, \widetilde{r}^{\varepsilon}(t)\right), t, \widetilde{r}^{\varepsilon}(t)\right) \\
& \times g\left(\varphi^{\varepsilon}, t, r^{\varepsilon}(t)\right)-\widetilde{g}^{T}\left(\varphi^{\varepsilon}, t, \widetilde{r}^{\varepsilon}(t)\right)
\end{aligned}
$$

$$
\begin{aligned}
& \times V_{x x}\left(\varphi^{\varepsilon}(0)-D\left(\varphi^{\varepsilon}, \widetilde{r}^{\varepsilon}(t)\right), t, \widetilde{r}^{\varepsilon}(t)\right) \\
& \left.\times \widetilde{g}\left(\varphi^{\varepsilon}, t, \widetilde{r}^{\varepsilon}(t)\right)\right] \\
& +\sum_{i=1}^{M} \widehat{\gamma}_{r^{\varepsilon}(t) i} \widetilde{V}\left(\varphi^{\varepsilon}(0)-D\left(x_{t}^{\varepsilon}, i\right), t, i\right) \\
& -\sum_{k=1}^{l} \tilde{\gamma}_{\widetilde{r}^{\varepsilon}}(t) k\left(\varphi^{\varepsilon}(0)-D\left(x_{t}^{\varepsilon}, k\right), t, k\right) \\
& =\mathscr{L} V\left(\varphi^{\varepsilon}, t, \widetilde{r}^{\varepsilon}(t)\right) \\
& +V_{x}\left(\varphi^{\varepsilon}(0)-D\left(\varphi^{\varepsilon}, \widetilde{r}^{\varepsilon}(t)\right), t, \widetilde{r}^{\varepsilon}(t)\right) \\
& \times\left[f\left(\varphi^{\varepsilon}, t, r^{\varepsilon}(t)\right)-\widetilde{f}\left(\varphi^{\varepsilon}, t, \widetilde{r}^{\varepsilon}(t)\right)\right] \\
& +\frac{1}{2} \operatorname{trace}\left[g^{T}\left(\varphi^{\varepsilon}, t, r^{\varepsilon}(t)\right)\right. \\
& \times V_{x x}\left(\varphi^{\varepsilon}(0)-D\left(\varphi^{\varepsilon}, \widetilde{r}^{\varepsilon}(t)\right), t, \widetilde{r}^{\varepsilon}(t)\right) \\
& \times g\left(\varphi^{\varepsilon}, t, r^{\varepsilon}(t)\right)-\tilde{g}^{T}\left(\varphi^{\varepsilon}, t, \widetilde{r}^{\varepsilon}(t)\right) \\
& \times V_{x x}\left(\varphi^{\varepsilon}(0)-D\left(\varphi^{\varepsilon}, \widetilde{r}^{\varepsilon}(t)\right), t, \widetilde{r}^{\varepsilon}(t)\right) \\
& \left.\times \widetilde{g}\left(\varphi^{\varepsilon}, t, \widetilde{r}^{\varepsilon}(t)\right)\right] \\
& +\sum_{i=1}^{M} \widehat{\gamma}_{r^{\varepsilon}(t) i} \widetilde{V}\left(\varphi^{\varepsilon}(0)-D\left(x_{t}^{\varepsilon}, i\right), t, i\right) \\
& -\sum_{k=1}^{l} \widetilde{\gamma}_{\bar{r}^{\varepsilon}(t) k} V\left(\varphi^{\varepsilon}(0)-D\left(x_{t}^{\varepsilon}, k\right), t, k\right) .
\end{aligned}
$$

Therefore,

$$
\begin{aligned}
U(t+h)-U(t) & \\
= & \limsup _{\varepsilon \rightarrow 0} \mathbb{E} \\
& \times \int_{t+\theta}^{t+\theta+h} e^{\gamma s} \\
& \times\left[\mathscr{L} V\left(\varphi^{\varepsilon}, s, \widetilde{r}^{\varepsilon}(s)\right)\right. \\
& \left.+\gamma V\left(\varphi^{\varepsilon}(0)-D\left(\varphi^{\varepsilon}, \widetilde{r}^{\varepsilon}(s)\right), s, \widetilde{r}^{\varepsilon}(s)\right)\right] d s \\
& +\limsup _{\varepsilon \rightarrow 0} \mathbb{E} \\
& \times \int_{t+\theta}^{t+\theta+h} e^{\gamma t} V_{x}\left(\varphi^{\varepsilon}(0)-D\left(\varphi^{\varepsilon}, \widetilde{r}^{\varepsilon}(s)\right), s, \widetilde{r}^{\varepsilon}(s)\right) \\
& \left.\left.\quad+\frac{1}{2} \limsup _{\varepsilon \rightarrow 0} \sup ^{\varepsilon}, s, r^{\varepsilon}(s)\right)-\widetilde{f}\left(\varphi^{\varepsilon}, s, \widetilde{r}^{\varepsilon}(s)\right)\right] d s \\
& \times \int_{t+\theta}^{t+\theta+h} e^{\gamma s}
\end{aligned}
$$




$$
\begin{aligned}
& \times \operatorname{trace}[ g^{T}\left(\varphi^{\varepsilon}, s, r^{\varepsilon}(s)\right) \\
& \times V_{x x}\left(\varphi^{\varepsilon}(0)-D\left(\varphi^{\varepsilon}, \widetilde{r}^{\varepsilon}(s)\right), s, \widetilde{r}^{\varepsilon}(s)\right) \\
& \times g\left(\varphi^{\varepsilon}, s, r^{\varepsilon}(s)\right)-\widetilde{g}^{T}\left(\varphi^{\varepsilon}, s, \widetilde{r}^{\varepsilon}(s)\right) \\
& \times V_{x x}\left(\varphi^{\varepsilon}(0)-D\left(\varphi^{\varepsilon}, \widetilde{r}^{\varepsilon}(s)\right), s, \widetilde{r}^{\varepsilon}(s)\right) \\
&\left.\times \widetilde{g}\left(\varphi^{\varepsilon}, s, \widetilde{r}^{\varepsilon}(s)\right)\right] d s \\
&+\limsup _{\varepsilon \rightarrow 0} \mathbb{E} \\
& \times \int_{t+\theta}^{t+\theta+h} e^{\gamma s} \\
& \times\left(\sum_{i=1}^{M} \widehat{\gamma}_{\gamma^{\varepsilon}}(s) i\right. \\
&
\end{aligned}
$$

By the definition of $\tilde{f}$,

$$
\begin{aligned}
f & \left(\varphi^{\varepsilon}, t, r^{\varepsilon}(t)\right)-\tilde{f}\left(\varphi^{\varepsilon}, t, \widetilde{r}^{\varepsilon}(t)\right) \\
& =\sum_{k=1}^{l} \sum_{j=1}^{M_{k}} f\left(\varphi^{\varepsilon}, t, s_{k j}\right) \times\left[I_{\left\{r^{\varepsilon}(t)=s_{k j}\right\}}-\mu_{j}^{k} I_{\left\{\tilde{r}^{\varepsilon}(t)=k\right\}}\right] .
\end{aligned}
$$

This implies that

$$
\begin{gathered}
\lim _{\varepsilon \rightarrow 0} \mathbb{E} \int_{t+\theta}^{t+\theta+h} e^{\gamma s} V_{x}\left(\varphi^{\varepsilon}(0)-D\left(\varphi^{\varepsilon}, \widetilde{r}^{\varepsilon}(s)\right), s, \widetilde{r}^{\varepsilon}(s)\right) \\
\times\left[f\left(\varphi^{\varepsilon}, s, r^{\varepsilon}(s)\right)-\widetilde{f}\left(\varphi^{\varepsilon}, s, \widetilde{r}^{\varepsilon}(s)\right)\right] d s \\
\leq \lim _{\varepsilon \rightarrow 0}\left[\mathbb{E} \mid \int_{t+\theta}^{t+\theta+h} e^{\gamma s} V_{x}\left(\varphi^{\varepsilon}(0)-D\left(\varphi^{\varepsilon}, \widetilde{r}^{\varepsilon}(s)\right), s, \widetilde{r}^{\varepsilon}(s)\right)\right. \\
\left.\times\left.\left[f\left(\varphi^{\varepsilon}, s, r^{\varepsilon}(s)\right)-\widetilde{f}\left(\varphi^{\varepsilon}, s, \widetilde{r}^{\varepsilon}(s)\right)\right] d s\right|^{2}\right]^{1 / 2} \\
=\lim _{\varepsilon \rightarrow 0}\left[\mathbb{E} \mid \int_{t+\theta}^{t+\theta+h} e^{\gamma s} V_{x}\left(\varphi^{\varepsilon}(0)-D\left(\varphi^{\varepsilon}, \widetilde{r}^{\varepsilon}(s)\right), s, \widetilde{r}^{\varepsilon}(s)\right)\right. \\
\times \sum_{k=1}^{l} \sum_{j=1}^{M_{k}} f\left(\varphi^{\varepsilon}, s, s_{k j}\right) \\
\left.\times\left.\left[I_{\left\{r^{\varepsilon}(s)=s_{k j}\right\}}-\mu_{j}^{k} I_{\left\{\tilde{r}^{\varepsilon}(s)=k\right\}}\right] d s\right|^{2}\right]^{1 / 2}
\end{gathered}
$$

$$
\begin{aligned}
\leq \lim _{\varepsilon \rightarrow 0}\left[\mathbb{E} \mid \int_{t+\theta}^{t+\theta+h} \sum_{k=1}^{l} \sum_{j=1}^{M_{k}} e^{\gamma s} L\left(1+\|\varphi\|^{p}\right)\right. \\
\left.\times\left.\left[I_{\left\{r^{\varepsilon}(s)=s_{k j}\right\}}-\mu_{j}^{k} I_{\left\{\tilde{r}^{\varepsilon}(s)=k\right\}}\right] d s\right|^{2}\right]^{1 / 2} .
\end{aligned}
$$

By the argument of Lemma 7.14 in [4], the right side of the above inequality is equivalent to 0 ; that is, $I_{2}=0$. Similarly, we can show that

$$
\begin{aligned}
I_{3}=\frac{1}{2} \limsup _{\varepsilon \rightarrow 0} \mathbb{E} & \\
\times \int_{t+\theta}^{t+\theta+h} e^{\gamma s} & \\
\times \operatorname{trace} & {\left[g^{T}\left(\varphi^{\varepsilon}, s, r^{\varepsilon}(s)\right)\right.} \\
& \times V_{x x}\left(\varphi^{\varepsilon}(0)-D\left(\varphi^{\varepsilon}, \widetilde{r}^{\varepsilon}(s)\right), s, \widetilde{r}^{\varepsilon}(s)\right) \\
& \times g\left(\varphi^{\varepsilon}, s, r^{\varepsilon}(s)\right) \\
& -\widetilde{g}^{T}\left(\varphi^{\varepsilon}, s, \widetilde{r}^{\varepsilon}(s)\right) \\
& \times V_{x x}\left(\varphi^{\varepsilon}(0)-D\left(\varphi^{\varepsilon}, \widetilde{r}^{\varepsilon}(s)\right), s, \widetilde{r}^{\varepsilon}(s)\right) \\
& \left.\times \widetilde{g}\left(\varphi^{\varepsilon}, s, \widetilde{r}^{\varepsilon}(s)\right)\right] d s=0 .
\end{aligned}
$$

By the definition of $\widehat{\Gamma}$ and $\widetilde{\Gamma}$, we have

$$
\begin{aligned}
& \sum_{i=1}^{M} \widehat{\gamma}_{r^{\varepsilon}(t) i} \widetilde{V}\left(\varphi^{\varepsilon}(0)-D\left(x_{t}^{\varepsilon}, i\right), t, i\right) \\
& =\widehat{\Gamma} \widetilde{V}\left(\varphi^{\varepsilon}(0)-D\left(x_{t}^{\varepsilon}, i\right), t, \cdot\right)\left(r^{\varepsilon}(t)\right), \\
& \sum_{k=1}^{l} \widetilde{\gamma}_{\widetilde{r}^{\varepsilon}(t) k} V\left(\varphi(0)-D\left(x_{t}^{\varepsilon}, k\right), t, k\right) \\
& =\widetilde{\Gamma} V\left(\varphi(0)-D\left(x_{t}^{\varepsilon}, k\right), t, \cdot\right)\left(\widetilde{r}^{\varepsilon}(t)\right) .
\end{aligned}
$$

Hence,

$$
\begin{aligned}
& I_{4}=\limsup _{\varepsilon \rightarrow 0} \mathbb{E} \\
& \times \int_{t+\theta}^{t+\theta+h} e^{\gamma s} \\
& \times\left(\sum_{i=1}^{M} \widehat{\gamma}_{r^{\varepsilon}(s) i} \widetilde{V}\left(\varphi^{\varepsilon}(0)-D\left(x_{s}^{\varepsilon}, i\right), s, i\right)\right. \\
& \left.-\sum_{k=1}^{l} \widetilde{\gamma}_{\hat{r}^{\varepsilon}(s) k} V\left(\varphi^{\varepsilon}(0)-D\left(x_{s}^{\varepsilon}, k\right), s, k\right)\right) d s
\end{aligned}
$$




$$
\begin{aligned}
& =\limsup _{\varepsilon \rightarrow 0} \mathbb{E} \\
& \times \int_{t+\theta}^{t+\theta+h} e^{\gamma s} \\
& \times\left(\widehat{\Gamma} \widetilde{V}\left(\varphi^{\varepsilon}(0)-D\left(x_{s}^{\varepsilon}, i\right), s, \cdot\right)\left(r^{\varepsilon}(s)\right)\right. \\
& \left.-\widetilde{\Gamma} V\left(\varphi^{\varepsilon}(0)-D\left(x_{s}^{\varepsilon}, k\right), s, \cdot\right)\left(s_{k j}\right)\right) d s \\
& =\limsup _{\varepsilon \rightarrow 0} \mathbb{E} \\
& \times \int_{t+\theta}^{t+\theta+h} e^{\gamma s} \sum_{k=1}^{l} \sum_{j=1}^{M_{k}} \widehat{\Gamma} \widetilde{V}\left(\varphi^{\varepsilon}(0)-D\left(x_{s}^{\varepsilon}, i\right), s, \cdot\right)\left(s_{k j}\right) \\
& \times\left[I_{\left\{r^{\varepsilon}(s)=s_{k j}\right\}}-\mu_{j}^{k} I_{\left\{\widetilde{r}^{\varepsilon}(s)=k\right\}}\right] d s \\
& \leq \limsup _{\varepsilon \rightarrow 0}\left[\mathbb{E} \mid \int_{t+\theta}^{t+\theta+h} e^{\gamma s}\right. \\
& \times \sum_{k=1}^{l} \sum_{j=1}^{M_{k}} \widehat{\Gamma} \widetilde{V}\left(\varphi^{\varepsilon}(0)-D\left(x_{s}^{\varepsilon}, i\right), s, \cdot\right)\left(s_{k j}\right) \\
& \left.\times\left.\left[I_{\left\{r^{\varepsilon}(s)=s_{k j}\right\}}-\mu_{j}^{k} I_{\left\{\tilde{r}^{\varepsilon}(s)=k\right\}}\right]\right|^{2}\right]^{1 / 2} .
\end{aligned}
$$

By the argument of Lemma 7.14 in [4], the right side of the above inequality is equivalent to 0 ; that is, $I_{4}=0$. Therefore,

$$
\begin{aligned}
& U(t+h)-U(t) \\
& =\lim _{\varepsilon \rightarrow 0} \mathbb{E} \\
& \quad \times \int_{t+\theta}^{t+\theta+h} e^{\gamma s} \\
& \quad \times\left[\mathscr{L} V\left(\varphi^{\varepsilon}, s, \widetilde{r}^{\varepsilon}(s)\right)\right. \\
& \left.\quad+\gamma V\left(\varphi^{\varepsilon}(0)-D\left(\varphi^{\varepsilon}, \widetilde{r}^{\varepsilon}(s)\right), s, \widetilde{r}^{\varepsilon}(s)\right)\right] d s
\end{aligned}
$$$$
\leq 0 \text {. }
$$

That is

$$
U(t+h) \leq U(t) .
$$

So, $U(t+h)=U(t)$ for all $h>0$ sufficiently small, and hence $D^{+} U(t)=0$. Inequality (34) holds.
It follows from (34) that $U(t) \leq U(0)$ for all $t \geq 0$. By the definition of $U(t)$,

$$
\begin{aligned}
& \limsup _{\varepsilon \rightarrow 0} e^{\gamma t} \mathbb{E}\left|x^{\varepsilon}(t)-D\left(x_{t}^{\varepsilon}, \widetilde{r}^{\varepsilon}(t)\right)\right|^{p} \\
& \leq c_{2} \limsup _{\varepsilon \rightarrow 0} \sup _{\tau \leq \theta \leq 0} e^{\gamma \theta} \mathbb{E}\left|x^{\varepsilon}(\theta)-D\left(x_{\theta}^{\varepsilon}, \widetilde{r}^{\varepsilon}(\theta)\right)\right|^{p} \\
& \leq c_{2} \limsup _{\varepsilon \rightarrow 0} \sup _{-\tau \leq \theta \leq 0}(1+\kappa)^{p-1} \\
& \quad \times\left[\mathbb{E}\left|x^{\varepsilon}(\theta)\right|^{p}+\kappa^{1-p} \mathbb{E}\left|D\left(x_{\theta}^{\varepsilon}, \widetilde{r}^{\varepsilon}(\theta)\right)\right|^{p}\right] \\
& \leq c_{2}(1+\kappa)^{p}\|\xi\|^{p}, \quad t \geq 0 .
\end{aligned}
$$

By Lemma 6, we derive

$$
\limsup _{\varepsilon \rightarrow 0} e^{\gamma t} \mathbb{E}\left|x^{\varepsilon}(t)\right|^{p} \leq \frac{c_{2}(1+\kappa)^{p}}{c_{1}(1-\kappa)^{p}}\|\xi\|^{p} .
$$

That is,

$$
\limsup _{\varepsilon \rightarrow 0} \mathbb{E}\left|x^{\varepsilon}(t)\right|^{p} \leq \frac{c_{2}(1+\kappa)^{p}}{c_{1}(1-\kappa)^{p}}\|\xi\|^{p} e^{-\gamma t}, \quad \forall t \geq 0 .
$$

\section{Neutral Stochastic Functional System with Pure Jump}

In this section, we discuss the stability of the following neutral stochastic functional system with pure jump:

$$
\begin{array}{r}
d\left[\varphi^{\varepsilon}(0)-D\left(\varphi^{\varepsilon}, r^{\varepsilon}(t)\right)\right] \\
=f\left(\varphi^{\varepsilon}, t, r^{\varepsilon}(t)\right) d t+\int_{\mathbb{R}^{m}} b\left(x_{t-}^{\varepsilon}, t, r^{\varepsilon}(t), z\right) \widetilde{N}(d t, d z) \\
x_{0}=\xi \in C\left([-\tau, 0] ; \mathbb{R}^{n}\right), \quad r(0) \in \mathbb{S},
\end{array}
$$

where $x_{t-}^{\varepsilon}=\lim _{s \uparrow t} x_{s}^{\varepsilon}, D: C\left([-\tau, 0] ; \mathbb{R}^{n}\right) \times \mathbb{S} \rightarrow \mathbb{R}^{n}, b:$ $C\left([-\tau, 0] ; \mathbb{R}^{n}\right) \times \mathbb{R}_{+} \times \mathbb{S} \times \mathbb{R}^{m} \rightarrow \mathbb{R}^{n \times m}$. We assume that each column $b^{(\delta)}$ of the $n \times m$ matrix $b=\left[b_{i j}\right]$ depends on $z$ only through the $\delta$ th coordinate $z_{\delta}$; that is,

$$
\begin{aligned}
& b^{(\delta)}(\varphi, t, i, z)=b^{(\delta)}\left(\varphi, t, i, z_{\delta}\right) \\
& z=\left(z_{1}, \ldots, z_{m}\right) \in \mathbb{R}^{m}, \quad i \in \mathbb{S} .
\end{aligned}
$$

$N(t, z)$ is an $m$-dimensional Poisson process and the compensated Poisson process is defined by

$$
\begin{aligned}
\widetilde{N}(d t, d z)= & \left(\widetilde{N}_{1}\left(d t, d z_{1}\right), \ldots, \widetilde{N}_{m}\left(d t, d z_{m}\right)\right) \\
= & \left(N_{1}\left(d t, d z_{1}\right)-\lambda_{1}\left(d z_{1}\right) d t, \ldots, N_{m}\left(d t, d z_{m}\right)\right. \\
& \left.-\lambda_{m}\left(d z_{m}\right) d t\right),
\end{aligned}
$$

where $\left\{N_{\delta}, \delta=1, \ldots, m\right\}$ are independent one-dimensional Poisson random measures with characteristic measure 
$\left\{\lambda_{\delta}, \delta=1, \ldots, m\right\}$ coming from $m$ independent onedimensional Poisson point processes. The limit system of (56) is defined as follows:

$$
\begin{aligned}
& d\left[\varphi^{\varepsilon}(0)-D\left(\widetilde{x}_{t}^{\varepsilon}, \widetilde{r}^{\varepsilon}(t)\right)\right] \\
& =\tilde{f}\left(\widetilde{x}_{t}^{\varepsilon}, t, \widetilde{r}^{\varepsilon}(t)\right) d t \\
& \quad+\int_{\mathbb{R}^{m}} \widetilde{b}\left(\widetilde{x}_{t-}^{\varepsilon}, t, \widetilde{r}^{\varepsilon}(t), z\right) \widetilde{N}(d t, d z) \\
& \widetilde{x}_{0}=\xi \in C\left([-\tau, 0] ; \mathbb{R}^{n}\right), \quad \widetilde{r}(0) \in \mathbb{S},
\end{aligned}
$$

where $\widetilde{x}_{t-}^{\varepsilon}=\lim _{s \uparrow t} \widetilde{x}_{s}^{\varepsilon}$ and $\widetilde{b}: C\left([-\tau, 0] ; \mathbb{R}^{n}\right) \times \mathbb{R}_{+} \times \widetilde{\mathbb{S}} \times \mathbb{R}^{m} \rightarrow$ $\mathbb{R}^{n \times m}$. Similar to the definition of $\tilde{f}$, we define

$$
\begin{gathered}
\widetilde{D}(\varphi, k)=\sum_{j=1}^{N_{m}} \mu_{j}^{k} D\left(\varphi, s_{k j}\right), \\
\widetilde{b}(\varphi, t, k, z)=\sum_{j=1}^{N_{m}} \mu_{j}^{k} b\left(\varphi, t, s_{k j}, z\right),
\end{gathered}
$$

for each $s_{k j} \in \mathbb{S}^{k}$ with $k \in\{1, \ldots, l\}$ and $j \in\left\{1, \ldots, N_{m}\right\}$.

To assure the existence and uniqueness of the solution of (59), we also give the following standard assumptions.

Assumption 8. For any integer $\zeta$, there is a constant $L_{\zeta}>0$, such that

$$
\begin{aligned}
& |f(\varphi, t, i)-f(\phi, t, i)| \\
& \quad \vee \sum_{\delta=1}^{m} \int_{\mathbb{R}}\left|b^{(\delta)}\left(\varphi, t, i, z_{\delta}\right)-b^{(\delta)}\left(\phi, t, i, z_{\delta}\right)\right| \lambda_{\delta}\left(d z_{\delta}\right) \\
& \quad \leq L_{\zeta}\|\varphi-\phi\|^{2},
\end{aligned}
$$

for all $i \in \mathbb{S}$ and those $\varphi, \phi \in C\left([-\tau, 0] ; \mathbb{R}^{n}\right)$ with $\|\varphi\| \vee\|\phi\| \leq \zeta$, and $f(0, t, i) \equiv 0, b(0, t, i, z) \equiv 0$.

Assumption 9. There is an $\bar{L}>0$, such that, for any $\varphi, \phi \in$ $C\left([-\tau, 0] ; \mathbb{R}^{n}\right), i \in \mathbb{S}$,

$$
\begin{aligned}
|f(\varphi, t, i)| & \vee \sum_{\delta=1}^{m} \int_{\mathbb{R}}\left|b^{(\delta)}\left(\varphi, t, i, z_{\delta}\right)\right| \lambda_{\delta}\left(d z_{\delta}\right) \\
& \leq \bar{L}\left(1+\|\varphi\|^{2}\right) .
\end{aligned}
$$

Assumption 10. For all $i \in \mathbb{S}$ and those $\varphi, \phi \in C\left([-\tau, 0] ; \mathbb{R}^{n}\right)$, there is a constant $0<\kappa<1$ such that

$$
\begin{gathered}
|D(\varphi, i)-D(\phi, i)| \leq \kappa\|\varphi-\phi\|^{2}, \\
D(0, i) \equiv 0 .
\end{gathered}
$$

Given that $V \in C^{p}\left(\mathbb{R}^{n} \times \mathbb{R}_{+} \times \mathbb{S} ; \mathbb{R}_{+}\right)$, define an operator $\mathbb{L} V$ by

$$
\begin{aligned}
& \mathbb{L} V(\varphi, t, i) \\
& =V_{t}(\varphi(0)-D(\varphi, i), t, i) \\
& +V_{x}(\varphi(0)-D(\varphi, i), t, i) f(\varphi, t, i) \\
& +\sum_{j=1}^{N} \gamma_{i j} V(\varphi(0)-D(\varphi, i), t, j) \\
& +\int_{\mathbb{R}} \sum_{\delta=1}^{m}\left\{V\left(\varphi(0)-D(\varphi, i)+b^{(\delta)}\left(\varphi, t, \iota, z_{\delta}\right), t, \iota\right)\right. \\
& -V(\varphi(0)-D(\varphi, i), t, i) \\
& -V_{x}(\varphi(0)-D(\varphi, i), t, i) \\
& \left.\times b^{(\delta)}\left(\varphi, t, \iota, z_{\delta}\right)\right\} \lambda_{\delta}\left(d z_{\delta}\right) \text {, }
\end{aligned}
$$

where

$$
\begin{gathered}
V_{x}(\varphi(0)-D(\varphi, i), t, i) \\
=\left(\frac{\partial V(\varphi(0)-D(\varphi, i), t, i)}{\partial x_{1}}, \ldots,\right. \\
\left.\frac{\partial V(\varphi(0)-D(\varphi, i), t, i)}{\partial x_{m}}\right) .
\end{gathered}
$$

Lemma 11 (see [20]). Let Assumptions 1, 8, and 9 hold, as $\varepsilon \rightarrow 0$; then, $\left(x^{\varepsilon}(\cdot), \widetilde{r}^{\varepsilon}(\cdot)\right)$ converges weakly to $(\widetilde{x}(\cdot), \widetilde{r}(\cdot))$ in $D\left([0, \infty), \mathbb{R}^{n} \times \widetilde{\mathbb{S}}\right)$, where $D\left([0, \infty), \mathbb{R}^{n} \times \widetilde{\mathbb{S}}\right)$ is the space of functions defined on $[0, \infty)$ that are right continuous and have left limits taking values in $\mathbb{R}^{n} \times \widetilde{\mathbb{S}}$ and are endowed with the Skorohod topology.

Theorem 12. Let Assumptions 1 and 8-10 hold and let $c_{1}, c_{2}$, $\lambda, p$ be all positive numbers and $q>1$. Assume that there exists a function $V(x, t, k) \in C^{p}\left(\mathbb{R}^{n} \times \mathbb{R}_{+} \times \widetilde{\mathbb{S}} ; \mathbb{R}_{+}\right)$satisfying Assumption 5, such that

$$
c_{1}|x|^{p} \leq V(x, t, k) \leq c_{2}|x|^{p}, \quad k \in \widetilde{\mathbb{S}},
$$

for all $(x, t, k) \in \mathbb{R}^{n} \times \mathbb{R}_{+} \times \widetilde{\mathbb{S}}$ and $t \geq 0, k \in \widetilde{\mathbb{S}}$. Consider the following:

$$
\begin{aligned}
& \mathbb{E}|D(\varphi, k)|^{p} \leq \kappa^{p} \sup _{-\tau \leq \theta \leq 0} e^{\bar{\imath} \theta}\|\varphi\|^{p}, \\
& \kappa=\max \left\{\kappa_{1}, \ldots, \kappa_{k}\right\}, \quad \varphi \in L_{\mathscr{F}_{t}}^{p},
\end{aligned}
$$

for all $t \geq 0,0<\kappa_{\sigma}<1, \sigma=\{1, \ldots, k\}$, and

$$
\mathbb{E}\left[\max _{k \in \widetilde{\mathbb{S}}} \mathbb{L} V(\varphi, t, k)\right] \leq-\gamma \mathbb{E}\left[\max _{k \in \widetilde{\mathbb{S}}} V(\varphi(0)-D(\varphi, k))\right],
$$


provided $\varphi=\{\varphi(\theta):-\tau \leq \theta \leq 0\} \in L_{\mathscr{F}_{t}}^{p}\left([-\tau, 0] ; \mathbb{R}^{n}\right)$, satisfying

$$
\begin{aligned}
\mathbb{E} & {\left[\min _{k \in \widetilde{\mathbb{S}}} V(x(t+\theta), t+\theta, k)\right] } \\
& <q \mathbb{E}\left[\max _{k \in \widetilde{\mathbb{S}}} V(\varphi(0)-D(\varphi, k), t, i)\right], \quad-\tau \leq \theta \leq 0 .
\end{aligned}
$$

Then, for all $\xi \in C\left([-\tau, 0] ; \mathbb{R}^{n}\right), t \geq 0$,

$$
\limsup _{\varepsilon \rightarrow 0} \mathbb{E}\left|x^{\varepsilon}(t)\right|^{p} \leq \frac{c_{2}(1+\kappa)^{p}}{c_{1}(1-\kappa)^{p}}\|\xi\|^{p} e^{-\bar{v} t},
$$

where

$$
\bar{\nu}=\min \left\{\bar{\gamma}, \frac{1}{\tau} \log \frac{q}{\left(c_{2} / c_{1}\right)(1-\kappa)^{p}}\right\},
$$

$\bar{\gamma}$ being the root of the following equation:

$$
\frac{c_{2}}{c_{1}}(1-\kappa)^{p} e^{\bar{\gamma} \tau}=\lambda .
$$

Proof. Define

$$
\widetilde{V}(\varphi, t, \rho)=\sum_{k=1}^{l} V(\varphi, t, k) I_{\left\{\rho \in \mathbb{S}^{k}\right\}}=V(\varphi, t, k), \quad \text { if } \rho \in \mathbb{S}^{k} .
$$

Extend $r(t)$ to $[-\tau, 0]$ by setting $r(t)=r(0)$; then, $\mathbb{E} V(x(t), t, r(t))$ is right continuous on $t \geq-\tau$. Let $\gamma \in(0, \bar{\nu})$ be arbitrary and define

$U(t)$

$$
\begin{gathered}
:=\sup _{-\tau \leq \theta \leq 0}\left[e ^ { \gamma ( t + \theta ) } \mathbb { E } V \left(x^{\varepsilon}(t+\theta)-D\left(x_{t+\theta}^{\varepsilon}, \widetilde{r}^{\varepsilon}(t+\theta)\right),\right.\right. \\
\left.\left.t+\theta, \widetilde{r}^{\varepsilon}(t+\theta)\right)\right] \\
=\sup _{-\tau \leq \theta \leq 0}\left[e ^ { \gamma ( t + \theta ) } \mathbb { E } \widetilde { V } \left(x^{\varepsilon}(t+\theta)-D\left(x_{t+\theta}^{\varepsilon}, r^{\varepsilon}(t+\theta)\right),\right.\right. \\
\left.\left.t+\theta, r^{\varepsilon}(t+\theta)\right)\right],
\end{gathered}
$$

for all $t \geq 0$. We claim that

$$
D^{+} U(t)=\limsup _{h \rightarrow 0+} \frac{U(t+h)-U(t)}{h} \leq 0, \quad \forall t \geq 0 .
$$

Similar to the proof of Theorem 7, we derive

$$
\begin{aligned}
\mathbb{E} V & \left(x^{\varepsilon}(t+\theta), t+\theta, \widetilde{r}^{\varepsilon}(t+\theta)\right) \\
& <q \mathbb{E} V\left(\varphi(0)-D\left(\varphi^{\varepsilon}, \widetilde{r}^{\varepsilon}(t)\right), t, \widetilde{r}^{\varepsilon}(t)\right),
\end{aligned}
$$

for all $-\tau \leq \theta \leq 0$, where $q>\left(c_{2} / c_{1}\right)(1-\kappa)^{p} e^{\gamma \tau}$; that is, $\gamma<$ $(1 / \tau)\left(\log \left(q /\left(c_{2} / c_{1}\right)(1-\kappa)^{p}\right)\right)$.
Thus,

$$
\begin{aligned}
& \mathbb{E}\left[\max _{k \in \widetilde{\mathbb{S}}} \mathbb{L} V\left(\varphi^{\varepsilon}, t, \widetilde{r}^{\varepsilon}(t)\right)\right] \\
& \quad \leq-\lambda \mathbb{E}\left[\max _{k \in \widetilde{\mathbb{S}}} V\left(\varphi^{\varepsilon}(0)-D\left(\varphi^{\varepsilon}, \widetilde{r}^{\varepsilon}(t)\right), t, \widetilde{r}^{\varepsilon}(t)\right)\right],
\end{aligned}
$$

which implies that

$$
\begin{aligned}
\mathbb{E} & {\left[\mathbb{L} V\left(\varphi^{\varepsilon}, t, \widetilde{r}^{\varepsilon}(t)\right)\right] } \\
& \leq-\lambda \mathbb{E}\left[V\left(\varphi^{\varepsilon}(0)-D\left(\varphi^{\varepsilon}, \widetilde{r}^{\varepsilon}(t)\right), t, \widetilde{r}^{\varepsilon}(t)\right)\right] .
\end{aligned}
$$

By the condition of $\gamma<\bar{\nu} \leq \lambda$, we get

$$
\begin{aligned}
\mathbb{E} & {\left[\mathbb{L} V\left(\varphi^{\varepsilon}, t, \widetilde{r}^{\varepsilon}(t)\right)\right] } \\
& \leq-\gamma \mathbb{E}\left[V\left(\varphi^{\varepsilon}(0)-D\left(\varphi^{\varepsilon}, \widetilde{r}^{\varepsilon}(t)\right), t, \widetilde{r}^{\varepsilon}(t)\right)\right] .
\end{aligned}
$$

We now consider

$$
\begin{aligned}
& U(t+h)-U(t) \\
& =\limsup _{\varepsilon \rightarrow 0}\left[e^{\gamma(t+\theta+h)} \mathbb{E}\right. \\
& \times\left[V \left(x^{\varepsilon}(t+\theta+h)\right.\right. \\
& -D\left(x_{t+\theta+h}^{\varepsilon}, \widetilde{r}^{\varepsilon}(t+\theta+h)\right), \\
& \left.\left.t+\theta+h, \widetilde{r}^{\varepsilon}(t+\theta+h)\right)\right] \\
& -e^{\gamma(t+\theta)} \mathbb{E} \\
& \times\left[V \left(x^{\varepsilon}(t+\theta)-D\left(x_{t+\theta}^{\varepsilon}, \widetilde{r}^{\varepsilon}(t+\theta)\right),\right.\right. \\
& \left.\left.\left.t+\theta, \widetilde{r}^{\varepsilon}(t+\theta)\right)\right]\right] \\
& =\limsup _{\varepsilon \rightarrow 0} \mathbb{E} \\
& \times \int_{t+\theta}^{t+\theta+h} e^{\gamma s}\left[\mathbb{L} V\left(\varphi^{\varepsilon}, s, \widetilde{r}^{\varepsilon}(s)\right)\right. \\
& \left.+\gamma V\left(\varphi^{\varepsilon}(0)-D\left(\varphi^{\varepsilon}, \widetilde{r}^{\varepsilon}(s)\right), s, \widetilde{r}^{\varepsilon}(t)\right)\right] d s \\
& =\limsup _{\varepsilon \rightarrow 0} \mathbb{E} \\
& \times \int_{t+\theta}^{t+\theta+h} e^{\gamma s} \\
& \times\left[\mathbb{L} \widetilde{V}\left(\varphi^{\varepsilon}, s, r^{\varepsilon}(s)\right)\right. \\
& \left.+\gamma V\left(\varphi^{\varepsilon}(0)-D\left(\varphi^{\varepsilon}, \widetilde{r}^{\varepsilon}(t)\right), s, \widetilde{r}^{\varepsilon}(s)\right)\right] d t .
\end{aligned}
$$


By the definition of the operator $\mathbb{L}$, we have

$$
\begin{aligned}
& \mathbb{L} \widetilde{V}\left(\varphi^{\varepsilon}, t, r^{\varepsilon}(t)\right) \\
& =\widetilde{V}_{t}\left(\varphi^{\varepsilon}(0)-D\left(\varphi^{\varepsilon}, r^{\varepsilon}(t)\right), t, r^{\varepsilon}(t)\right) \\
& +\widetilde{V}_{x}\left(\varphi^{\varepsilon}(0)-D\left(\varphi^{\varepsilon}, r^{\varepsilon}(t)\right), t, r^{\varepsilon}(t)\right) \\
& \quad \times f\left(\varphi^{\varepsilon}, t, r^{\varepsilon}(t)\right) \\
& +\sum_{\delta=1}^{m} \int_{\mathbb{R}}\left\{\widetilde { V } \left(\varphi^{\varepsilon}(0)-D\left(\varphi^{\varepsilon}, r^{\varepsilon}(t)\right)\right.\right. \\
& \left.+b^{(\delta)}\left(x_{t-}^{\varepsilon}, t, r^{\varepsilon}(t), z_{\delta}\right), t, r^{\varepsilon}(t)\right) \\
& -\widetilde{V}^{\varepsilon}\left(\varphi^{\varepsilon}(0)-D\left(\varphi^{\varepsilon}, r^{\varepsilon}(t)\right), t, r^{\varepsilon}(t)\right) \\
& -\widetilde{V}_{x}\left(\varphi^{\varepsilon}(0)-D\left(\varphi^{\varepsilon}, r^{\varepsilon}(t)\right), t, r^{\varepsilon}(t)\right) \\
& \left.\quad \times b^{(\delta)}\left(x_{t-}^{\varepsilon}, t, r^{\varepsilon}(t), z_{\delta}\right)\right\} \lambda_{\delta}\left(d z_{\delta}\right) \\
& +\sum_{j=1}^{N} \gamma_{r^{\varepsilon}(t) j}^{\varepsilon}\left(\varphi^{\varepsilon}(0)-D\left(\varphi^{\varepsilon}, j\right), t, j\right) \\
& =\mathbb{L} V\left(\varphi^{\varepsilon}, t, \widetilde{r}^{\varepsilon}(t)\right) \\
& +V_{x}\left(\varphi^{\varepsilon}(0)-D\left(\varphi^{\varepsilon}, \widetilde{r}^{\varepsilon}(t)\right), t, \widetilde{r}^{\varepsilon}(t)\right) \\
& +\left[f\left(\varphi^{\varepsilon}, t, r^{\varepsilon}(t)\right)-\widetilde{f}\left(\varphi^{\varepsilon}, t, \widetilde{r}^{\varepsilon}(t)\right)\right] \\
& +\sum_{\delta=1}^{m} \int_{\mathbb{R}}\left\{V \left(\varphi^{\varepsilon}(0)-D\left(\varphi^{\varepsilon}, \widetilde{r}^{\varepsilon}(t)\right)\right.\right. \\
& \left.+b^{(\delta)}\left(x_{t-}^{\varepsilon}, t, \widetilde{r}^{\varepsilon}(t), z_{\delta}\right), t, \widetilde{r}^{\varepsilon}(t)\right)
\end{aligned}
$$$$
\times \lambda_{\delta}\left(d z_{\delta}\right)
$$$$
-\sum_{\delta=1}^{m} \int_{\mathbb{R}}\left\{V _ { x } \left(\varphi^{\varepsilon}(0)\right.\right.
$$$$
\left.-D\left(\varphi^{\varepsilon}, \widetilde{r}^{\varepsilon}(t)\right), t, \widetilde{r}^{\varepsilon}(t)\right)
$$$$
\times\left(b^{(\delta)}\left(x_{t-}^{\varepsilon}, t, r^{\varepsilon}(t), z_{\delta}\right)\right.
$$$$
\left.\left.-\widetilde{b}^{(\delta)}\left(x_{t-}^{\varepsilon}, t, \widetilde{r}^{\varepsilon}(t), z_{\delta}\right)\right)\right\} \lambda_{\delta}\left(d z_{\delta}\right)
$$$$
+\sum_{j=1}^{N} \widehat{\gamma}_{\gamma^{\varepsilon}(t) j} \widetilde{V}\left(\varphi^{\varepsilon}(0)-D\left(\varphi^{\varepsilon}, j\right), t, j\right)
$$$$
-\sum_{k=1}^{l} \widetilde{\gamma}_{\tilde{r}^{\varepsilon}}(t) k\left(\varphi^{\varepsilon}(0)-D\left(\varphi^{\varepsilon}, k\right), t, k\right) .
$$

This implies that

$$
\begin{aligned}
& U(t+h)-U(t) \\
& =\lim \sup \mathbb{E} \\
& \times \int_{t+\theta}^{t+\theta+h} e^{\gamma s} \\
& \times\left[\llbracket V\left(\varphi^{\varepsilon}, s, \widetilde{r}^{\varepsilon}(s)\right)\right. \\
& +\gamma V\left(\varphi^{\varepsilon}(0)\right. \\
& \left.\left.-D\left(\varphi^{\varepsilon}, \widetilde{r}^{\varepsilon}(s)\right), s, \widetilde{r}^{\varepsilon}(s)\right)\right] d s \\
& +\limsup _{\varepsilon \rightarrow 0} \mathbb{E} \\
& \times \int_{t+\theta}^{t+\theta+h} e^{\gamma s} \\
& \times V_{x}\left(\varphi^{\varepsilon}(0)-D\left(\varphi^{\varepsilon}, \widetilde{r}^{\varepsilon}(s)\right), s, \widetilde{r}^{\varepsilon}(s)\right) \\
& \times\left[f\left(\varphi^{\varepsilon}, s, r^{\varepsilon}(s)\right)-\tilde{f}\left(\varphi^{\varepsilon}, s, \widetilde{r}^{\varepsilon}(s)\right)\right] d s \\
& +\limsup _{\varepsilon \rightarrow 0} \mathbb{E} \\
& \times \int_{t+\theta}^{t+\theta+h} e^{\gamma s} \\
& \times\left[\sum _ { \delta = 1 } ^ { m } \int _ { \mathbb { R } } \left[V \left(\varphi^{\varepsilon}(0)-D\left(\varphi^{\varepsilon}, \widetilde{r}^{\varepsilon}(s)\right)\right.\right.\right. \\
& \left.+b^{(\delta)}\left(x_{s^{-}}^{\varepsilon}, s, \widetilde{r}^{\varepsilon}(s), z_{\delta}\right), s, \widetilde{r}^{\varepsilon}(s)\right) \\
& -V\left(\varphi^{\varepsilon}(0)-D\left(\varphi^{\varepsilon}, \widetilde{r}^{\varepsilon}(s)\right)\right. \\
& +\widetilde{b}^{(\delta)}\left(x_{s^{-}}^{\varepsilon}, s, \widetilde{r}^{\varepsilon}(s), z_{\delta}\right), \\
& \left.\left.s, \widetilde{r}^{\varepsilon}(s)\right)\right] \\
& \left.\times \lambda_{\delta}\left(d z_{\delta}\right)\right] d s \\
& -\limsup _{\varepsilon \rightarrow 0} \mathbb{E} \\
& \times \int_{t+\theta}^{t+\theta+h} e^{\gamma s} \\
& \times\left\{\sum _ { \delta = 1 } ^ { m } \int _ { \mathbb { R } } \left[V_{x}\left(\varphi^{\varepsilon}(0)-D\left(\varphi^{\varepsilon}, \widetilde{r}^{\varepsilon}(s)\right), s, \widetilde{r}^{\varepsilon}(s)\right)\right.\right. \\
& \times\left(b^{(\delta)}\left(x_{s^{-}}^{\varepsilon}, s, r^{\varepsilon}(s), z_{\delta}\right)\right. \\
& \left.\left.-\widetilde{b}^{(\delta)}\left(x_{s^{-}}^{\varepsilon}, s, \widetilde{r}^{\varepsilon}(s), z_{\delta}\right)\right)\right] \\
& \left.\times \lambda_{\delta}\left(d z_{\delta}\right)\right\} d s
\end{aligned}
$$




$$
\begin{aligned}
& +\limsup _{\varepsilon \rightarrow 0} \mathbb{E} \\
& \times \int_{t+\theta}^{t+\theta+h} e^{\gamma s} \\
& \times\left(\sum_{j=1}^{N} \widehat{\gamma}_{r^{\varepsilon}(s) j} \widetilde{V}\left(\varphi^{\varepsilon}(0)-D\left(\varphi^{\varepsilon}, j\right), s, j\right)\right. \\
& \left.-\sum_{k=1}^{l} \tilde{\gamma}_{\tilde{r}^{\varepsilon}(s) k}\left(\varphi^{\varepsilon}(0)-D\left(\varphi^{\varepsilon}, k\right), s, k\right)\right) d s \\
& =: J_{1}+J_{2}+J_{3}+J_{4}+J_{5} \text {. }
\end{aligned}
$$

By the definition of $\widetilde{b}$,

$$
\begin{aligned}
b^{(\delta)}\left(x_{t-}^{\varepsilon}, t, r^{\varepsilon}(t), z_{\delta}\right)-\widetilde{b}^{(\delta)}\left(x_{t-}^{\varepsilon}, t, \widetilde{r}^{\varepsilon}(t), z_{\delta}\right) \\
=\sum_{i=1}^{l} \sum_{j=1}^{N_{k}} b^{(\delta)}\left(x_{t-}^{\varepsilon}, t, s_{k j}, z_{\delta}\right) \\
\quad \times\left[I_{\left\{r^{\varepsilon}(t)=s_{k j}\right\}}-\mu_{j}^{k} I_{\left\{\widetilde{r}^{\varepsilon}(t)=k\right\}}\right] .
\end{aligned}
$$

By Assumption 8, we have

$$
\begin{aligned}
& J_{4}=\limsup _{\varepsilon \rightarrow 0} \sum_{\delta=1}^{m} \mathbb{E} \\
& \times \int_{t+\theta}^{t+\theta+h} e^{\gamma s} \\
& \times V_{x}\left(\varphi^{\varepsilon}(0)-D\left(\varphi^{\varepsilon}, \widetilde{r}^{\varepsilon}(s)\right), s, \widetilde{r}^{\varepsilon}(s)\right) \\
& \times \int_{\mathbb{R}}\left[b^{(\delta)}\left(x_{s-}^{\varepsilon}, s, r^{\varepsilon}(s), z_{\delta}\right)\right. \\
& \left.-\widetilde{b}^{(\delta)}\left(x_{s-}^{\varepsilon}, s, \widetilde{r}^{\varepsilon}(s), z_{\delta}\right)\right] \\
& \times \lambda_{\delta}\left(d z_{\delta}\right) d s \\
& =\limsup _{\varepsilon \rightarrow 0} \sum_{\delta=1}^{m} \sum_{k=1}^{l} \sum_{j=1}^{N_{k}} \mathbb{E} \\
& \times \int_{t+\theta}^{t+\theta+h} e^{\gamma s} \\
& \times V_{x}\left(\varphi^{\varepsilon}(0)-D\left(\varphi^{\varepsilon}, \widetilde{r}^{\varepsilon}(s)\right),\right. \\
& \left.s, \widetilde{r}^{\varepsilon}(s)\right) \\
& \times \int_{\mathbb{R}} b^{(\delta)}\left(x_{s^{-}}^{\varepsilon}, s, s_{k j}, z_{\delta}\right) \\
& \times\left[I_{\left\{r^{\varepsilon}(s)=s_{k j}\right\}}\right. \\
& \left.-\mu_{j}^{k} I_{\left\{\hat{r}^{\tilde{}}(s)=k\right\}}\right] \\
& \times \lambda_{\delta}\left(d z_{\delta}\right) d s
\end{aligned}
$$

$$
\begin{aligned}
& \leq \limsup _{\varepsilon \rightarrow 0} \sum_{\delta=1}^{m} \sum_{k=1}^{l} \sum_{j=1}^{N_{k}}\left[\mathbb{E} \mid \int_{t+\theta}^{t+\theta+h}\right. e^{\gamma s} \\
& \times V_{x}\left(\varphi^{\varepsilon}(0)-D\left(\varphi^{\varepsilon}, \widetilde{r}^{\varepsilon}(s)\right),\right. \\
&\left.s, \widetilde{r}^{\varepsilon}(s)\right) \\
& \times \int_{\mathbb{R}} b^{(\delta)}\left(x_{s^{-}}^{\varepsilon}, s, s_{k j}, z_{\delta}\right) \\
& \times\left[I_{\left\{r^{\varepsilon}(s)=s_{k j}\right\}}\right. \\
&\left.\quad-\mu_{j}^{k} I_{\left\{\tilde{r}^{\varepsilon}(s)=k\right\}}\right] \\
& \times\left.\lambda_{\delta}\left(d z_{\delta}\right) d s\right|^{2} .
\end{aligned}
$$

By the argument of Lemma 7.14 in [4], the right side of the above inequality is equivalent to 0 ; that is, $J_{4}=0$. Similarly, by mean-value theorem, we can show that there exists $\eta_{t}^{(\delta)}$ which is between $\varphi^{\varepsilon}(0)-D\left(\varphi^{\varepsilon}, \widetilde{r}^{\varepsilon}(t)\right)+b^{(\delta)}\left(x_{t-}^{\varepsilon}, t, \widetilde{r}^{\varepsilon}(t), z_{\delta}\right)$ and $\varphi^{\varepsilon}(0)-D\left(\varphi^{\varepsilon}, \widetilde{r}^{\varepsilon}(t)\right)+\widetilde{b}^{(\delta)}\left(x_{t-}^{\varepsilon}, t, \widetilde{r}^{\varepsilon}(t), z_{\delta}\right)$ such that

$$
\begin{aligned}
& J_{3}=\lim _{\varepsilon \rightarrow 0} \sum_{\delta=1}^{m} \mathbb{E} \\
& \times \int_{t+\theta}^{t+\theta+h} e^{\gamma s}\left\{\int_{\mathbb{R}} V_{x}\left(\eta_{s}\right)\right. \\
& \times\left[b^{(\delta)}\left(x_{s^{-}}^{\varepsilon}, s, r^{\varepsilon}(s), z_{\delta}\right)\right. \\
& \left.-\widetilde{b}^{(\delta)}\left(x_{s-}^{\varepsilon}, s, \widetilde{r}^{\varepsilon}(s), z_{\delta}\right)\right] \\
& \left.\times \lambda_{\delta}\left(d z_{\delta}\right)\right\} d s \\
& =\lim _{\varepsilon \rightarrow 0} \sum_{\delta=1}^{m} \sum_{k=1}^{l} \sum_{j=1}^{N_{k}} \mathbb{E} \\
& \times \int_{t+\theta}^{t+\theta+h} e^{\gamma s} V_{x}\left(\eta_{s}\right) \\
& \times \int_{\mathbb{R}} b^{(\delta)}\left(x_{s-}^{\varepsilon}, s, s_{k j}, z_{\delta}\right) \\
& \times\left[I_{\left\{r^{\varepsilon}(s)=s_{k j}\right\}}-\mu_{j}^{k} I_{\left\{\tilde{r}^{\varepsilon}(s)=k\right\}}\right] \\
& \times \lambda_{\delta}\left(d z_{\delta}\right) d s \\
& \leq \lim _{\varepsilon \rightarrow 0} \sum_{\delta=1}^{m} \sum_{k=1}^{l} \sum_{j=1}^{N_{k}}\left[\mathbb{E} \mid \int_{t+\theta}^{t+\theta+h} e^{\gamma s} V_{x}\left(\eta_{t}\right)\right. \\
& \times \int_{\mathbb{R}} b^{(\delta)}\left(x_{s-}^{\varepsilon}, s, s_{k j}, z_{\delta}\right)
\end{aligned}
$$




$$
\begin{aligned}
& \times\left[I_{\left\{r^{\varepsilon}(s)=s_{k j}\right\}}-\mu_{j}^{k} I_{\left\{\tilde{r}^{\tilde{r}}(s)=k\right\}}\right] \\
& \left.\times\left.\lambda_{\delta}\left(d z_{\delta}\right) d s\right|^{2}\right]^{1 / 2} .
\end{aligned}
$$

By the argument of Lemma 7.14 in [4], we have $J_{3}=0$. Similar to the proof of Theorem 7, we derive $J_{2}=0, J_{5}=0$. Therefore, we arrive at

$$
\begin{aligned}
& U(t+h)-U(t) \\
& =\lim _{\varepsilon \rightarrow 0} \mathbb{E} \int_{t+\theta}^{t+\theta+h} e^{\gamma s} \\
& \times \quad\left[\mathbb{L V}\left(\varphi^{\varepsilon}, s, \widetilde{r}^{\varepsilon}(s)\right)\right. \\
& \left.\quad+\gamma V\left(\varphi^{\varepsilon}(0)-D\left(\varphi^{\varepsilon}, \widetilde{r}^{\varepsilon}(s)\right), s, \widetilde{r}^{\varepsilon}(s)\right)\right] d s
\end{aligned}
$$$$
\leq 0 .
$$

Then,

$$
U(t+h) \leq U(t) .
$$

Similar to the proof of Theorem 7, we get

$$
\mathbb{E}\left|x^{\varepsilon}(t)\right|^{p} \leq \frac{c_{2}(1+\kappa)^{p}}{c_{1}(1-\kappa)^{p}}\|\xi\|^{p} e^{-\bar{\nu} t} .
$$

The proof is therefore completed.

\section{Examples}

We will give two examples to illustrate our theory.

Example 1. Let $r^{\varepsilon}(\cdot)$ be a Markov chain generated by $\Gamma^{\varepsilon}$ given in (14) with

$$
\begin{aligned}
\bar{\Gamma} & =\left(\begin{array}{ccccc}
-1 & 0 & 1 & 0 & 0 \\
1 & -2 & 1 & 0 & 0 \\
2 & 1 & -3 & 0 & 0 \\
0 & 0 & 0 & -1 & 1 \\
0 & 0 & 0 & 1 & -1
\end{array}\right), \\
\widehat{\Gamma} & =\left(\begin{array}{ccccc}
-1 & 0 & 1 & 0 & 0 \\
0 & -1 & 0 & 1 & 0 \\
0 & 0 & -1 & 0 & 1 \\
0 & 1 & 0 & -1 & 0 \\
1 & 0 & 0 & 0 & -1
\end{array}\right)
\end{aligned}
$$

The generator $\bar{\Gamma}$ is made up of two irreducible blocks; by

$$
\left(\begin{array}{lll}
\pi_{1} & \pi_{2} & \pi_{3}
\end{array}\right)\left(\begin{array}{ccc}
-1 & 0 & 1 \\
1 & -2 & 1 \\
2 & 1 & -3
\end{array}\right)=0
$$

and $\pi_{1}+\pi_{2}+\pi_{3}=1$, we get $\mu^{1}=(5 / 8,1 / 8,1 / 4)$. In the same way, by

$$
\left(\begin{array}{ll}
\pi_{4} & \pi_{5}
\end{array}\right)\left(\begin{array}{cc}
-1 & 1 \\
1 & -1
\end{array}\right)=0
$$

and $\pi_{4}+\pi_{5}=1$, we have $\mu^{2}=(1 / 2,1 / 2)$. So,

$$
\begin{aligned}
\bar{\Gamma}=\bar{\mu} \widehat{\Gamma} \mathbf{1}=\left(\begin{array}{ccccc}
\frac{5}{8} & \frac{1}{8} & \frac{1}{4} & 0 & 0 \\
0 & 0 & 0 & \frac{1}{2} & \frac{1}{2}
\end{array}\right) \\
\\
\\
\times\left(\begin{array}{ccccc}
-1 & 0 & 1 & 0 & 0 \\
0 & -1 & 0 & 1 & 0 \\
0 & 0 & -1 & 0 & 1 \\
0 & 1 & 0 & -1 & 0 \\
1 & 0 & 0 & 0 & -1
\end{array}\right)\left(\begin{array}{ll}
1 & 0 \\
1 & 0 \\
1 & 0 \\
0 & 1 \\
0 & 1
\end{array}\right) \\
=\left(\begin{array}{cc}
-\frac{3}{8} & \frac{3}{8} \\
1 & -1
\end{array}\right) .
\end{aligned}
$$

Consider a one-dimensional neutral stochastic functional differential equation as follows:

$$
\begin{aligned}
& d\left[\varphi^{\varepsilon}(0)-D\left(\varphi^{\varepsilon}, r^{\varepsilon}(t)\right)\right] \\
& \quad=f\left(\varphi^{\varepsilon}, r^{\varepsilon}(t)\right) d t+g\left(\varphi^{\varepsilon}, r^{\varepsilon}(t)\right) d w(t),
\end{aligned}
$$

with

$$
\begin{gathered}
D\left(\varphi, s_{11}\right)=-0.6 \int_{-1}^{0} \varphi(\theta) d \theta, \\
D\left(\varphi, s_{12}\right)=-0.2 \int_{-1}^{0} \varphi(\theta) d \theta, \\
D\left(\varphi, s_{13}\right)=-0.4 \int_{-1}^{0} \varphi(\theta) d \theta, \\
f\left(\varphi, s_{11}\right)=-16 \varphi(0)-8 \cos [\varphi(0)], \\
f\left(\varphi, s_{12}\right)=8 \varphi(0)+4 \cos [\varphi(0)], \\
f\left(\varphi, s_{13}\right)=16 \varphi(0), \\
g\left(\varphi, s_{11}\right)=\frac{\sqrt{10}}{10} \int_{-1}^{0} \varphi(\theta) d \theta \cos \left[\int_{-1}^{0} \varphi(\theta) d \theta\right], \\
g\left(\varphi, s_{12}\right)=-\frac{\sqrt{2}}{2} \int_{-1}^{0} \varphi(\theta) d \theta \sin \left[\int_{-1}^{0} \varphi(\theta) d \theta\right], \\
g\left(\varphi, s_{13}\right)=\frac{\sqrt{3}}{2} \int_{-1}^{0} \varphi(\theta) d \theta, \\
D\left(\varphi, s_{21}\right)=0.5 \int_{-1}^{0} \varphi(\theta) d \theta, \\
D\left(\varphi, s_{22}\right)=0.5 \int_{-1}^{0} \varphi(\theta) d \theta, \\
f\left(\varphi, s_{21}\right)=-2 \varphi(0),
\end{gathered}
$$




$$
\begin{aligned}
& g\left(\varphi, s_{21}\right)=\frac{\int_{-1}^{0} \varphi(\theta) d \theta \sin \left[\int_{-1}^{0} \varphi(\theta) d \theta\right]}{4 \sqrt{2}}, \\
& g\left(\varphi, s_{22}\right)=\frac{\int_{-1}^{0} \varphi(\theta) d \theta \cos \left[\int_{-1}^{0} \varphi(\theta) d \theta\right]}{4 \sqrt{2}} .
\end{aligned}
$$

For any $\varphi \in L_{\mathscr{F}_{t}}^{2}([-1,0] ; \mathbb{R})$ and $\kappa=\max \{0.6,0.2,0.4\}=0.6$, applying the Hölder inequality yields

$$
\mathbb{E}|D(\varphi, i)|^{2} \leq 0.6^{2} \sup _{-1 \leq \theta \leq 0} e^{\nu \theta} \mathbb{E}\left|\int_{-1}^{0} \varphi(\theta) d \theta\right|^{2} \leq 0.36\|\varphi\|^{2},
$$

which implies condition (24). Then, the limit equation is

$$
d[\widetilde{\varphi}(0)-D(\widetilde{\varphi}, \widetilde{r}(t))]=f(\widetilde{\varphi}, \widetilde{r}(t)) d t+g(\widetilde{\varphi}, \widetilde{r}(t)) d w(t),
$$

where $\widetilde{r}$ is the Markov chain generated by $\widetilde{\Gamma}$ and

$$
\begin{gathered}
\widetilde{D}(\varphi, 1)=-0.5 \int_{-1}^{0} \varphi(\theta) d \theta, \\
\widetilde{D}(\varphi, 2)=0.5 \int_{-1}^{0} \varphi(\theta) d \theta, \\
\widetilde{f}(\varphi, 1)=-5 \varphi(0), \quad \widetilde{f}(\varphi, 2)=-2 \varphi(0), \\
\widetilde{g}(\varphi, 1)=\frac{1}{2} \int_{-1}^{0} \varphi(\theta) d \theta, \quad \widetilde{g}(\varphi, 2)=\frac{1}{4} \int_{-1}^{0} \varphi(\theta) d \theta .
\end{gathered}
$$

We define $V(x, 1)=2 x^{2}, V(x, 2)=x^{2}$. And by simple calculation, we can get

$$
\begin{gathered}
\mathscr{L} V(\varphi, 1) \leq-20 \frac{3}{8} \varphi^{2}(0)+\frac{13}{32}\left|\int_{-1}^{0} \varphi(\theta) d \theta\right|^{2}, \\
\mathscr{L} V(\varphi, 2) \leq-\frac{5}{2} \varphi^{2}(0)+\frac{13}{16}\left|\int_{-1}^{0} \varphi(\theta) d \theta\right|^{2} .
\end{gathered}
$$

Consequently,

$$
\begin{aligned}
\max _{i=1,2} \mathscr{L} V(\varphi, i) & \leq-\frac{5}{2} \varphi^{2}(0)+\frac{13}{16}\left|\int_{-1}^{0} \varphi(\theta) d \theta\right|^{2} \\
& =-\frac{5}{4}\left[\max _{i=1,2} V(x, i)\right]+\frac{13}{16}\left[\min _{i=1,2} V(x, i)\right] .
\end{aligned}
$$

It is easy to find a $q>1$ such that $5 / 4-13 q / 16>0$. Therefore, for any $\phi \in L_{\mathscr{F}_{t}}^{2}([-1,0] ; \mathbb{R})$ satisfying $\mathbb{E}\left[\min _{i \in \tilde{\mathbb{S}}} \phi(\theta)\right] \leq$ $q \mathbb{E}\left[\max _{i \in \widetilde{\mathbb{S}}} \phi(0)\right]$ on $-1 \leq \theta \leq 0$, (100) yields

$$
\mathbb{E}\left[\max _{i \in \widetilde{\mathbb{S}}} \mathscr{L} V(\varphi, i)\right] \leq-\left(\frac{5}{4}-\frac{13 q}{16}\right) \mathbb{E}\left[\max _{i=1,2} V(x, i)\right] .
$$

Hence, by Theorem 7 , the solution $x^{\varepsilon}(t)$ is mean square stable when $\varepsilon$ is sufficiently small.

Example 2. Let $r^{\varepsilon}(\cdot)$ be a Markov chain generated by

$$
\Gamma^{\varepsilon}=\frac{1}{\varepsilon} \widetilde{\Gamma}+\widehat{\Gamma}=\frac{1}{\varepsilon}\left(\begin{array}{cccc}
-2 & 0 & 2 & 0 \\
1 & -2 & 0 & 1 \\
0 & 2 & -2 & 0 \\
0 & 1 & 1 & -2
\end{array}\right) .
$$

Here, we set $\widehat{\Gamma}=0$. By a similar way, we get the stationary distribution $\mu=(2 / 11,4 / 11,3 / 11,2 / 11)$.

Consider the following one-dimensional equation:

$$
\begin{aligned}
d & {\left[\varphi^{\varepsilon}(0)-D\left(\varphi^{\varepsilon}, r^{\varepsilon}(t)\right)\right] } \\
& =f\left(\varphi^{\varepsilon}, r^{\varepsilon}(t)\right) d t+\int_{0}^{\infty} \sigma\left(r^{\varepsilon}(t), z\right) x_{t-}^{\varepsilon} \widetilde{N}(d t, d z)
\end{aligned}
$$

with

$$
\begin{gathered}
D(\varphi, 1)=-0.9 \int_{-1}^{0} \varphi(\theta) d \theta \\
D(\varphi, 2)=-0.4 \int_{-1}^{0} \varphi(\theta) d \theta \\
D(\varphi, 3)=-0.5 \int_{-1}^{0} \varphi(\theta) d \theta \\
D(\varphi, 4)=-0.3 \int_{-1}^{0} \varphi(\theta) d \theta \\
f(\varphi, 1)=2 \sin [\varphi(0)], \quad f(\varphi, 2)=-\frac{11}{2} \varphi(0), \\
f(\varphi, 3)=-\frac{11}{3} \varphi(0), \quad f(\varphi, 4)=-2 \sin [\varphi(0)] . \\
\text { Let } \quad \\
\alpha(z)=\frac{2}{11} \sigma(1, z)+\frac{4}{11} \sigma(2, z)+\frac{3}{11} \sigma(3, z)+\frac{2}{11} \sigma(4, z), \\
\int_{0}^{\infty} \alpha^{2}(z) \lambda(d z)<2 .
\end{gathered}
$$

For any $\varphi \in L_{\mathscr{F}_{t}}^{2}([-1,0] ; \mathbb{R})$ and $\kappa=\max \{0.9,0.4,0.5,0.3\}=$ 0.9 , applying the Hölder inequality yields

$$
\mathbb{E}|D(\varphi, i)|^{2} \leq 0.9^{2} \sup _{-1 \leq \theta \leq 0} e^{\bar{\nu} \theta} \mathbb{E}\left|\int_{-1}^{0} \varphi(\theta) d \theta\right|^{2} \leq 0.81\|\varphi\|^{2},
$$

which implies condition (67). Then, the limit equation is

$$
\begin{aligned}
& d\left[\varphi(0)+0.5 \int_{-1}^{0} \varphi(\theta) d \theta\right] \\
& \quad=-3 \varphi(0) d t+\int_{0}^{\infty} \alpha(z) \widetilde{x}_{t-} \widetilde{N}(d t, d z) .
\end{aligned}
$$


Let $V(x)=x^{2}$; then

$$
\mathbb{L} V(\varphi, i) \leq-6 \varphi^{2}(0)+\int_{0}^{\infty} \alpha^{2}(z) \lambda(d z)\left|\int_{-1}^{0} \varphi(\theta) d \theta\right|^{2} .
$$

We can find a $q>1$ such that $6-2 q>0$. Therefore, for any $\phi \in$ $L_{\mathscr{F}_{t}}^{2}([-1,0] ; \mathbb{R})$ satisfying $\mathbb{E}\left[\min _{i \in \widetilde{S}} \phi(\theta)\right] \leq q \mathbb{E}\left[\max _{i \in \widetilde{S}} \phi(0)\right]$ on $-1 \leq \theta \leq 0$, (108) yields

$$
\mathbb{E}\left[\max _{i \in \widetilde{\mathbb{S}}} \mathbb{L} V(\varphi, i)\right] \leq-(6-2 q) \mathbb{E}\left[\max _{i=1,2} V(x, i)\right] .
$$

Hence, by Theorem 12 , the solution $x^{\varepsilon}(t)$ is mean square stable.

\section{Conflict of Interests}

The authors declare that there is no conflict of interests regarding the publication of this paper.

\section{Acknowledgment}

This paper was supported by the National Science Foundation of China with Grant no. 61374085.

\section{References}

[1] Y. Shen and J. Wang, "Noise-induced stabilization of the recurrent neural networks with mixed time-varying delays and Markovian-switching parameters," IEEE Transactions on Neural Networks, vol. 18, no. 6, pp. 1857-1862, 2007.

[2] Y. Shen and J. Wang, "Almost sure exponential stability of recurrent neural networks with Markovian switching," IEEE Transactions on Neural Networks, vol. 20, no. 5, pp. 840-855, 2009.

[3] H. A. Simon and A. Ando, "Aggregation of variables in dynamic systems," Econometrica, vol. 29, pp. 111-138, 1961.

[4] G. G. Yin and Q. Zhang, Continuous-Time Markov Chains and Applications: A Singular Perturbations Approach, Springer, New York, NY, USA, 1998.

[5] H. Chen, C. Zhu, and Y. Zhang, "A note on exponential stability for impulsive neutral stochastic partial functional differential equations," Applied Mathematics and Computation, vol. 227, pp. 139-147, 2014.

[6] G. Hu and K. Wang, "Stability in distribution of neutral stochastic functional differential equations with Markovian switching," Journal of Mathematical Analysis and Applications, vol. 385, no. 2, pp. 757-769, 2012.

[7] S. Janković, M. Vasilova, and M. Krstić, "Some analytic approximations for neutral stochastic functional differential equations," Applied Mathematics and Computation, vol. 217, no. 8, pp. 36153623, 2010.

[8] V. Kolmanovskii, N. Koroleva, T. Maizenberg, X. Mao, and A. Matasov, "Neutral stochastic differential delay equations with Markovian switching," Stochastic Analysis and Applications, vol. 21, no. 4, pp. 819-847, 2003.

[9] D. Li and D. Xu, "Attracting and quasi-invariant sets of stochastic neutral partial functional differential equations," Acta Mathematica Scientia B, vol. 33, no. 2, pp. 578-588, 2013.
[10] X. Mao, "Exponential stability in mean square of neutral stochastic differential-functional equations," Systems \& Control Letters, vol. 26, no. 4, pp. 245-251, 1995.

[11] X. Mao, "Razumikhin-type theorems on exponential stability of neutral stochastic functional-differential equations," SIAM Journal on Mathematical Analysis, vol. 28, no. 2, pp. 389-401, 1997.

[12] Y. Song and Y. Shen, "New criteria on asymptotic behavior of neutral stochastic functional differential equations," Automatica, vol. 49, no. 2, pp. 626-632, 2013.

[13] F. Wu, S. Hu, and C. Huang, "Robustness of general decay stability of nonlinear neutral stochastic functional differential equations with infinite delay," Systems \& Control Letters, vol. 59, no. 3-4, pp. 195-202, 2010.

[14] S. Zhou and S. Hu, "Razumikhin-type theorems of neutral stochastic functional differential equations," Acta Mathematica Scientia B, vol. 29, no. 1, pp. 181-190, 2009.

[15] G. Badowski and G. G. Yin, "Stability of hybrid dynamic systems containing singularly perturbed random processes," IEEE Transactions on Automatic Control, vol. 47, no. 12, pp. 2021-2032, 2002.

[16] J. Hu, X. Mao, and C. Yuan, "Razumikhin-type theorems on exponential stability of SDDEs containing singularly perturbed random processes," Abstract and Applied Analysis, vol. 2013, Article ID 854743, 12 pages, 2013.

[17] A. A. Pervozvanskii and V. G. Gaitsgori, Theory of Suboptimal Decisions: Decomposition and Aggregation, Kluwer Academic, Dordrecht, The Netherlands, 1988.

[18] F. Wu, G. G. Yin, and L. Y. Wang, "Stability of a pure random delay system with two-time-scale Markovian switching," Journal of Differential Equations, vol. 253, no. 3, pp. 878-905, 2012.

[19] F. Wu, G. Yin, and L. Y. Wang, "Moment exponential stability of random delay systems with two-time-scale Markovian switching," Nonlinear Analysis: Real World Applications, vol. 13, no. 6, pp. 2476-2490, 2012.

[20] G. Yin and H. Yang, "Two-time-scale jump-diffusion models with Markovian switching regimes," Stochastics and Stochastics Reports, vol. 76, no. 2, pp. 77-99, 2004.

[21] C. Yuan and G. Yin, "Stability of hybrid stochastic delay systems whose discrete components have a large state space: a twotime-scale approach," Journal of Mathematical Analysis and Applications, vol. 368, no. 1, pp. 103-119, 2010.

[22] R. Z. Khasminskii, G. Yin, and Q. Zhang, "Asymptotic expansions of singularly perturbed systems involving rapidly fluctuating Markov chains," SIAM Journal on Applied Mathematics, vol. 56, no. 1, pp. 277-293, 1996.

[23] H. J. Kushner, Approximation and Weak Convergence Methods for Random Processes, with Applications to Stochastic Systems Theory, The MIT Press, Cambridge, Mass, USA, 1984. 


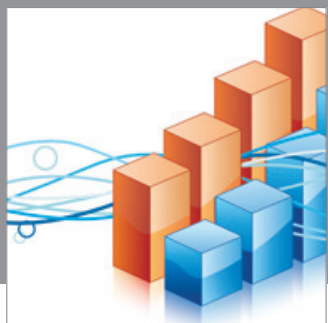

Advances in

Operations Research

mansans

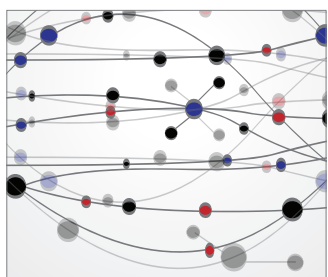

The Scientific World Journal
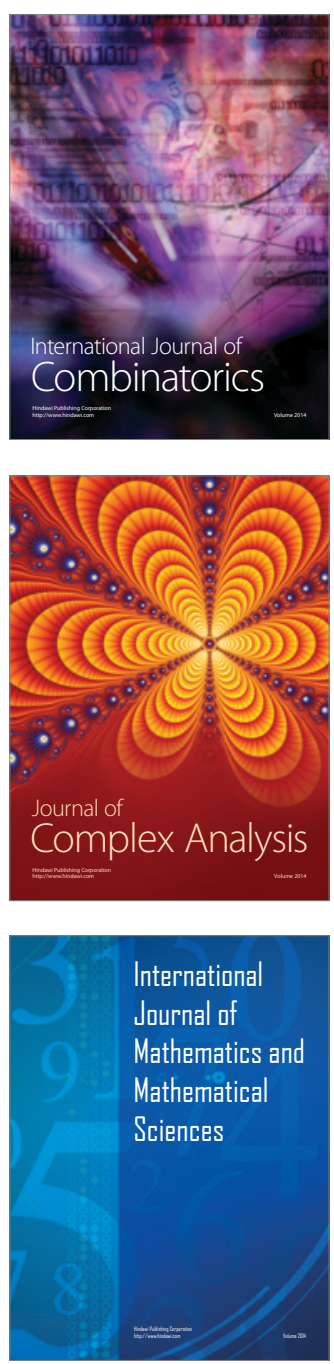
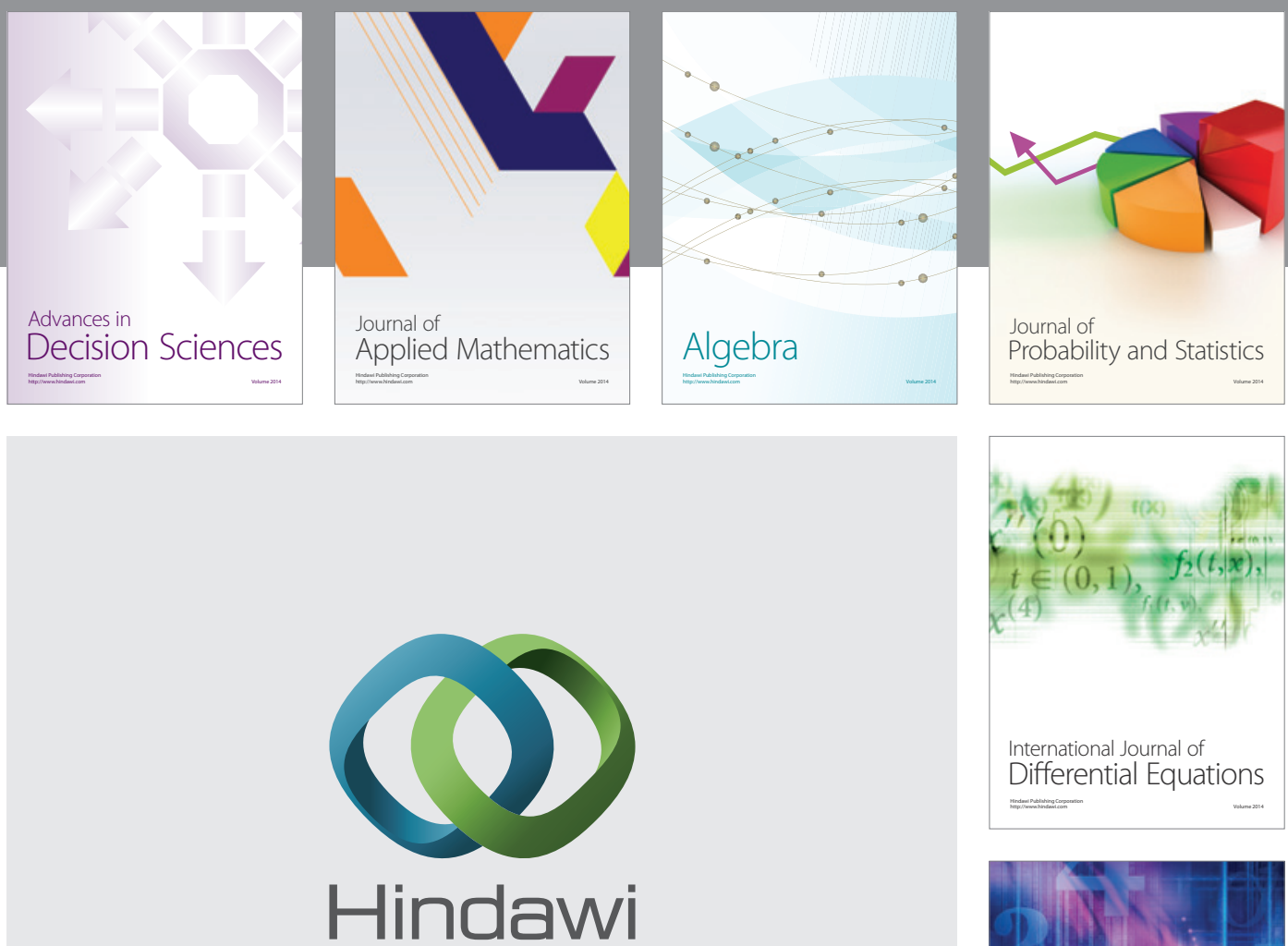

Submit your manuscripts at http://www.hindawi.com
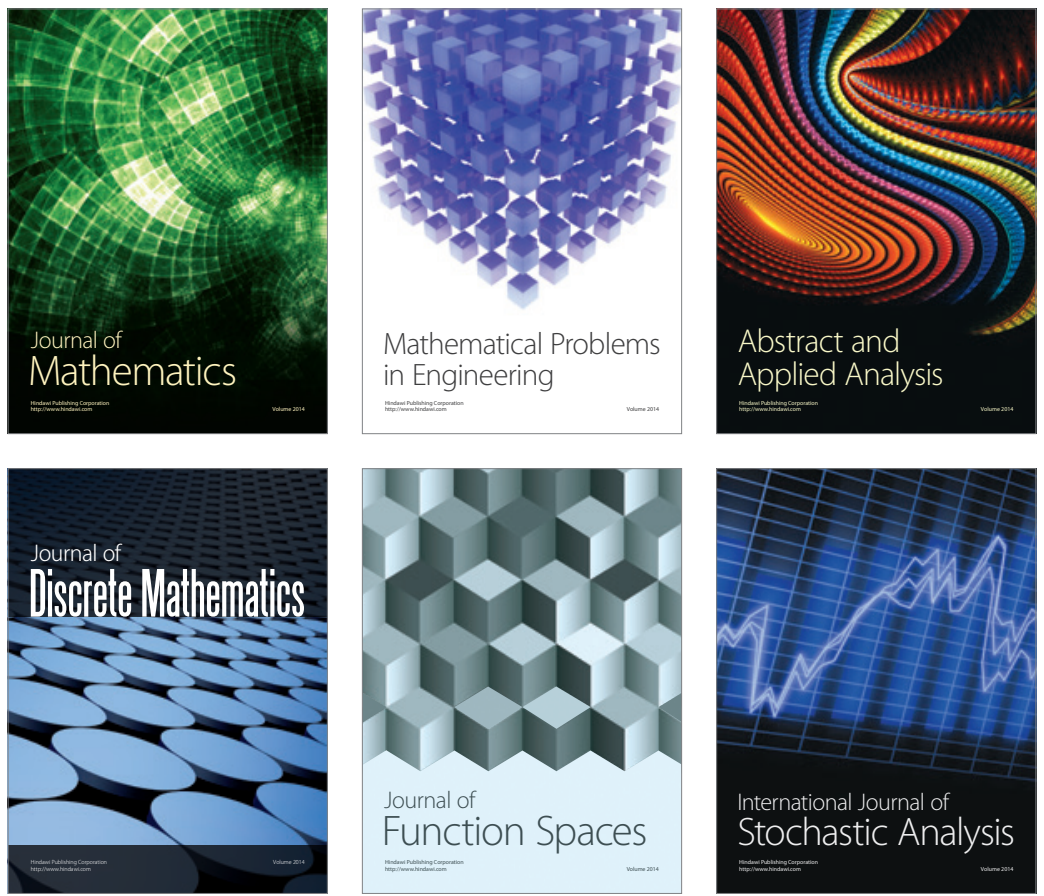

Journal of

Function Spaces

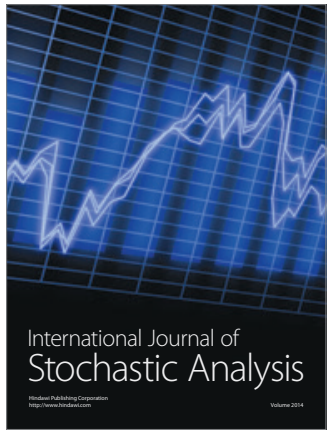

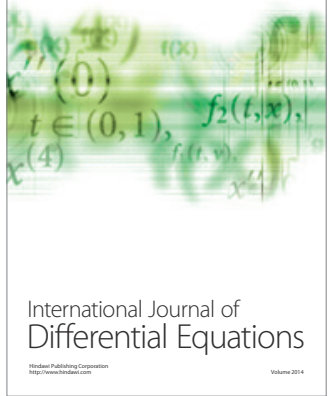
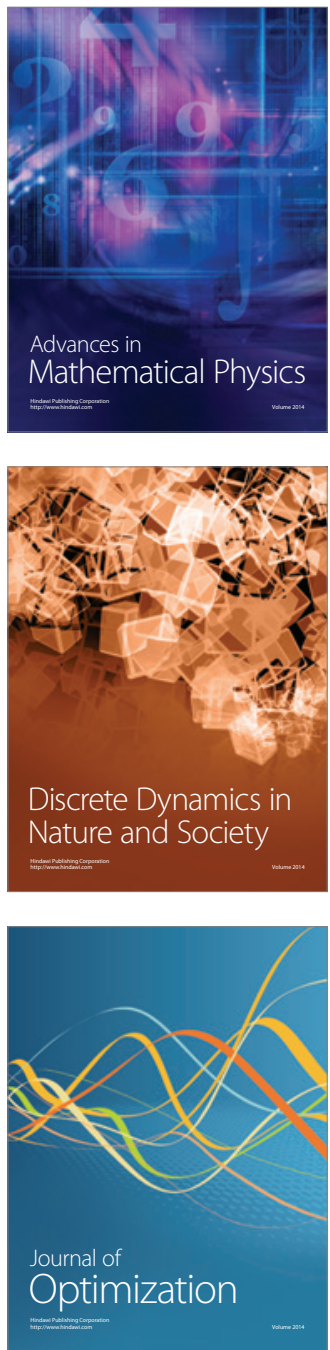